\title{
Science Readiness of the Gemini MCAO System: GeMS
}

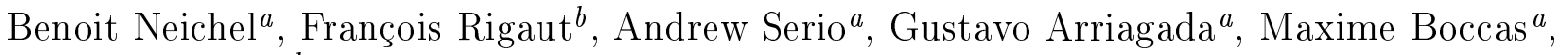 \\ Céline d'Orgeville ${ }^{b}$, Vincent Fesquet ${ }^{a}$, Chadwick Trujillo ${ }^{a}$, William N. Rambold ${ }^{a}$, Ramon L. \\ Galvez $^{a}$, Gaston Gausachs ${ }^{a}$, Tomislav B. Vucina ${ }^{a}$, Vanessa Montes ${ }^{a}$, Cristian Urrutia ${ }^{a}$, \\ Cristian Moreno ${ }^{a}$, Sarah J. Diggs ${ }^{a}$, Claudio Araya ${ }^{a}$, Javier Lührs ${ }^{a}$, Gelys Trancho ${ }^{c}$, Matthieu \\ Bec $^{c}$, Claudio Marchant ${ }^{a}$, Fabian Collao ${ }^{a}$, Eleazar R. Carrasco ${ }^{a}$, Michelle L. Edwards ${ }^{a}$, Peter \\ Pessev $^{a}$, Ariel Lopez ${ }^{a}$, Herman Diaz ${ }^{a}$ \\ ${ }^{a}$ Gemini Observatory, c/o AURA, Casilla 603 La Serena, Chile \\ ${ }^{b}$ Australian National University Research School of Astronomy and Astrophysics, Mount \\ Stromlo Observatory, Cotter Road, Weston ACT 2611, Australia \\ ${ }^{c}$ Giant Magellan Telescope Organization Corporation, PO box 90933, Pasadena, CA, \\ 91109-0933, USA
}

\begin{abstract}
The Gemini Multi-Conjugate Adaptive Optics System (GeMS) began its on-sky commissioning in January 2011. The system provides high order wide-field corrections using a constellation of five Laser Guide Stars. In December 2011, commissioning culminated in images with a FWHM of $80 \pm 2$ mas at 1.65 microns ( $\mathrm{H}$ band) over an $87 \mathrm{x}$ 87 arcsecond field of view. The first images have already demonstrated the scientific potential of GeMS, and after more than a year of commissioning GeMS is finally close to completion and ready for science. This paper presents a general status of the GeMS project and summarizes the achievements made during more than a year of commissioning. The characterization of GeMS performance is presented in a companion paper: "GeMS on-sky results", Rigaut et al. ${ }^{1}$ Here we report on the sub-systems' performance, discuss current limitations and present proposed upgrades. The integration of GeMS into the observatory operational scheme is detailed. Finally, we present the plans for next year's operations with GeMS.
\end{abstract}

Keywords: Multi-Conjugate Adaptive Optics Laser Guide Star, MCAO, LGS, GeMS

\section{INTRODUCTION TO GEMS}

GeMS is the Gemini Multi-Conjugated Adaptive Optics (MCAO) System. It is a facility instrument for the Gemini-South telescope, delivering a uniform, diffraction-limited Near-Infrared (NIR) image over an extended FoV of 2arcmin. The main sub-systems of GeMS are the Laser Guide Star Facility (LGSF) and the AO bench (Canopus). The characteristics of these systems are discussed below. A more detailed description can be found in previous presentations ${ }^{2-6}$ and on the GeMS public website: http://www.gemini.edu/sciops/instruments/gems.

GeMS works with a Laser Guide Star (LGS) constellation much like that of the 5-spots on dice: 4 of the LGS spots are on the edges of a 60 arcsec square, with the 5 th positioned in the center. These LGSs are produced by a $50 \mathrm{~W}$ laser split into 5 distinct 10-Watt beacons by a series of beamsplitters. The laser bench and its electronics enclosure are housed inside a Laser Service Enclosure ${ }^{7}$ (LSE), an $8 \times 2 \mathrm{~m}$ clean room built in-house on an extension of the telescope elevation platform (a Nasmyth focus for other telescopes, see Fig.1). The LSE ensures cleanliness and temperature stability with a requirement of $\pm 0.5 \mathrm{deg}^{\circ} \mathrm{C} / \mathrm{hr}$ and $\pm 2 \mathrm{deg}^{\circ} \mathrm{C}$ year round. Temperature stability is crucial to minimize laser cavity mis-alignment and optimize the laser performance. The Beam Transfer Optics (BTO), a subsystem of the LGSF, relays the laser beam(s) from the output of the Laser system to the input of the Laser Launch Telescope (LLT) located behind the telescope secondary mirror. Fig.1 illustrates this optical path. Beside relaying the laser light from the laser to the LLT, other BTO functionalities include constellation alignment

Further author information: (Send correspondence to bneichel@gemini.edu or frigaut@mso.anu.edu.au)

Adaptive Optics Systems III, edited by Brent L. Ellerbroek, Enrico Marchetti, Jean-Pierre Véran, Proc. of SPIE Vol. 8447, 84474 Q ( ) 2012 SPIE $\cdot$ CCC code: $0277-786 / 12 / \$ 18 \cdot$ doi: $10.1117 / 12.925285$ 
control, slow and fast compensation of telescope flexures, laser beam quality monitoring, beam shuttering, and laser polarization control. More details about the LGSF and its on-sky performance are presented in a companion paper. $^{8}$
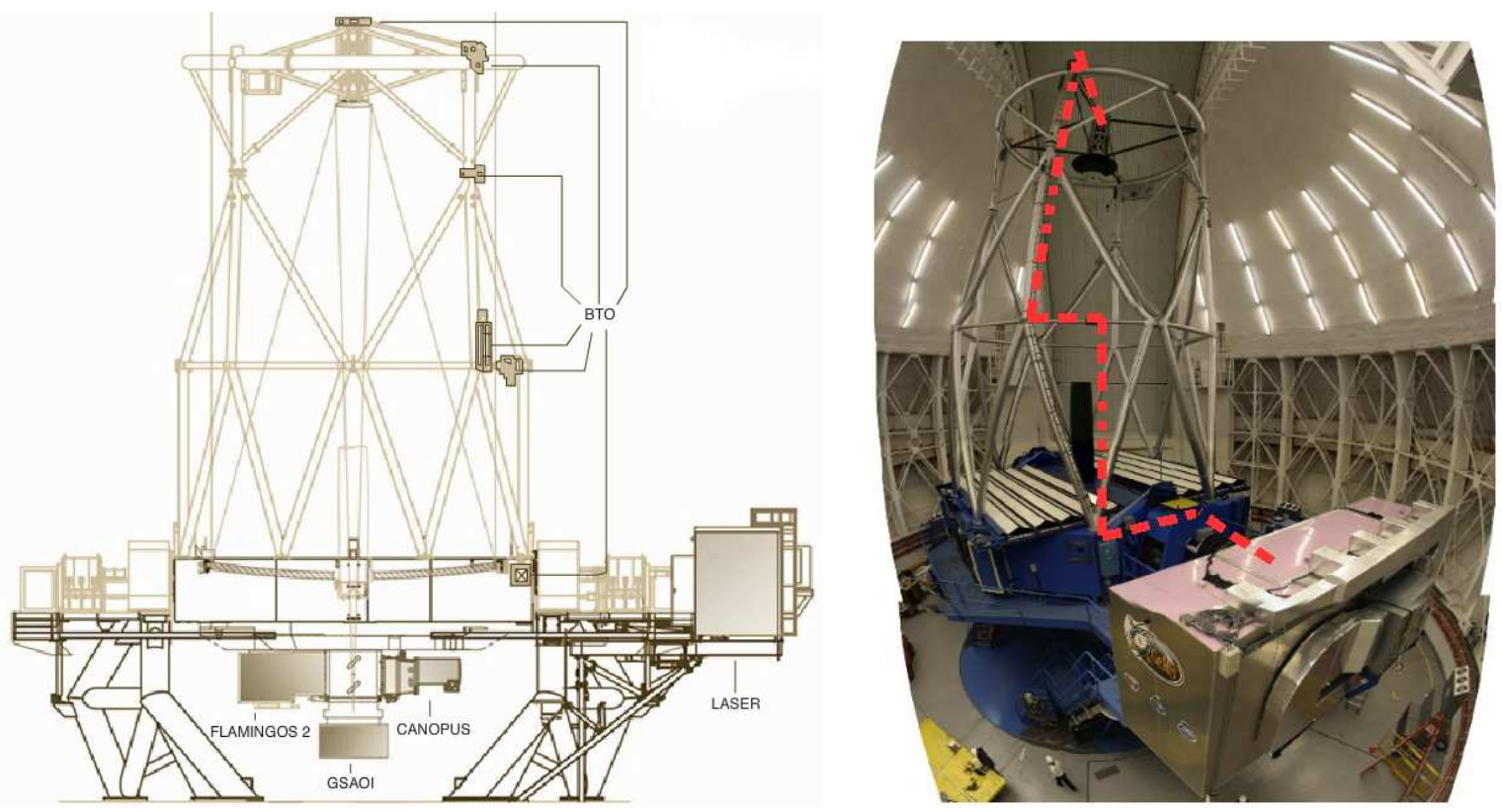

Figure 1. . Left: Schematic view of the Gemini Telescope and the GeMS components. Right: Picture of the actual installation of GeMS. The BTO path has been superimposed in orange.

The F/16 beam of the Gemini telescope passes through the Cassegrain Instrument Support Structure (ISS) and is re-directed to the Canopus bench (Fig.2) via the AO-fold mirror

The MCAO correction is then performed by three Deformable Mirrors (DMs) conjugated to 0, 4.5 and $9 \mathrm{~km}$ and one Tip-Tilt Mirror (TTM). After this, a first dichroic beam splitter is responsible for separating the visible from NIR light, sending the former to the WaveFront Sensors (WFS) and the latter to the science output with a $\mathrm{F} / 33.2$ focal-ratio to feed the instruments.

Science path. Currently, three instruments can use the corrected beam: GSAOI, ${ }^{9}$ FlamingosII ${ }^{10}$ and GMOS. ${ }^{11}$ The science instruments provide an On-Instrument (OI) guiding capability that is used to compensate for differential flexure that exists between the AO bench and the instruments themselves.

Wave-Front Sensor path. Within the AO bench, the visible light directed toward the WFS is split into a narrow range around $589 \mathrm{~nm}$ to illuminate the five LGS Wave-Front Sensors (LGSWFS); the remaining wavelengths go to the Natural Guide Star WFS (NGSWFS). The LGSWFS is composed of five 16x16 superature Shack-Hartmann lenslet arrays. They provide 2040 slope measurements, used to compute the MCAO high-order correction applied at a rate of up to $800 \mathrm{~Hz}$ by the three DMs. The NGSWFS is made of 3 probes, each containing a reflective pyramid that acts like a quad-cell feeding a set of 4 fibers + APDs. Fig. 3 shows a schematic view of this design. All of this is integrated into a small arm about $8 \mathrm{~mm}$ in size. Finally, one of the probes contains a small beam splitter that sends $30 \%$ of the light to a Slow Focus Sensor (SFS).

Two modes of operations will be offered with GeMS. The first mode (called $3+1$ ), uses the 3 visible pyramid probes described above combined with 1 OI star to correct for flexure. The second mode is specific to GSAOI, as this instrument can also provide a fast Tip-Tilt guiding capability. This is based on an On-Detector Guide Window (ODGWs) technology. ${ }^{12}$ The ODGWs are used in a fast readout mode, providing the Tip-Tilt information based on the centroid position of the stars. In this mode, one visible star (one of the three probes) is still needed 


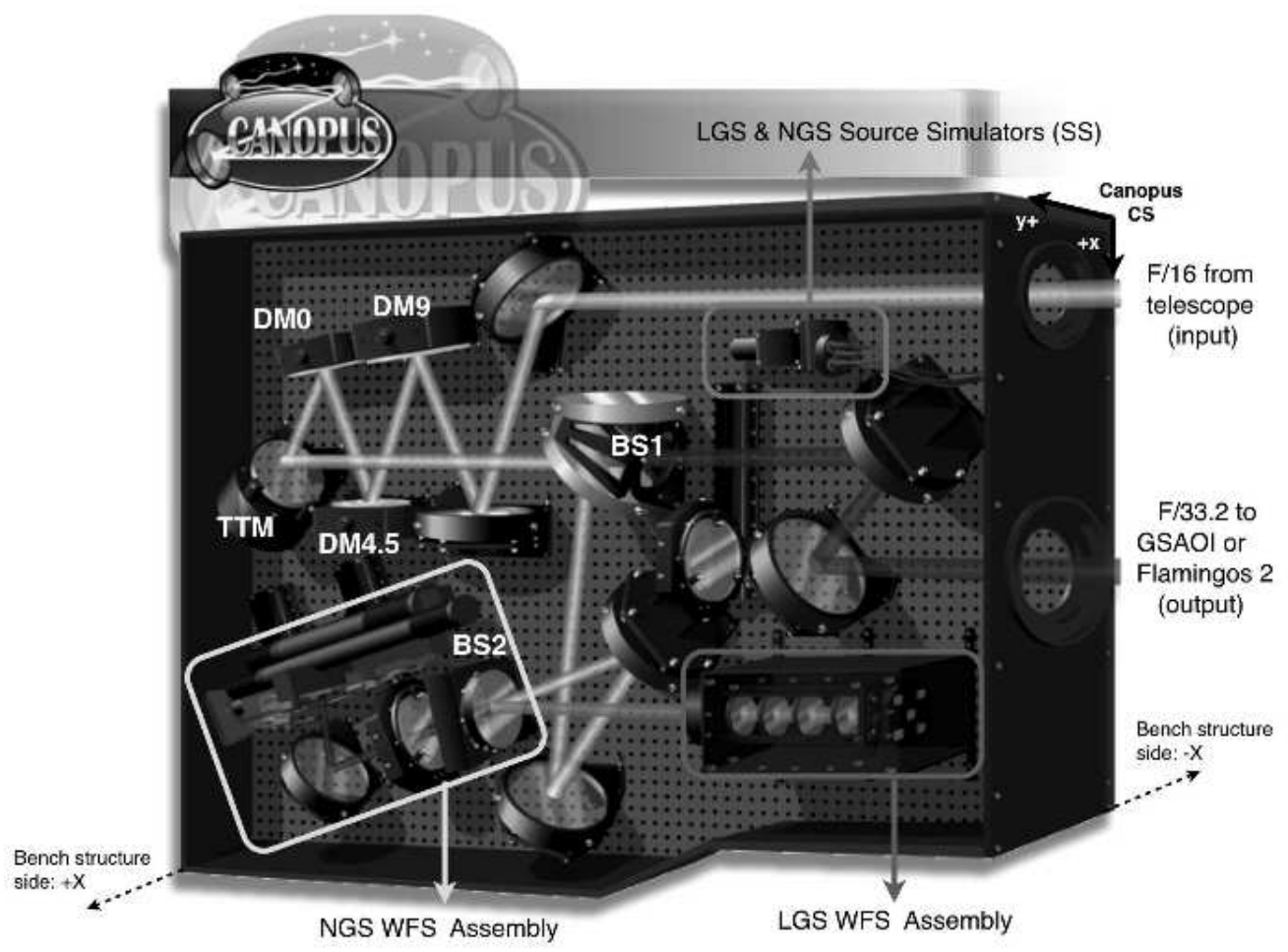

Figure 2. Schematic view of the Canopus MCAO bench.

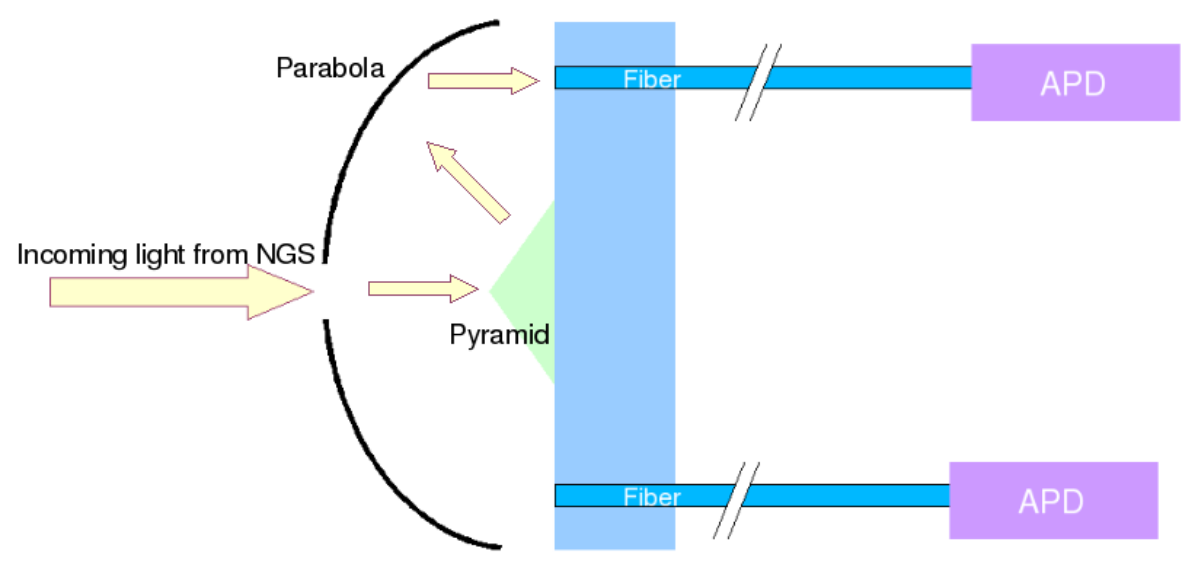

Figure 3. Simplified cross-section schematic of the current NGSWFS design. The light enters the module from the left.

for slow focus compensation. This mode is called $1+3$, i.e., one visible focus NGS and three fast near-infrared Tip-Tilt NGSs.

\subsection{One year of commissioning in a nutshell}

In early October 2010, the decision was made to move Canopus to the telescope and start on-sky commissioning as soon as possible. This decision was motivated by the seasonal weather conditions at Cerro Pachon and the need for clear nights to propagate the laser for efficient commissioning. This anticipated transportation accelerated the ending of the Integration and Test (IT) phase, and set the beginning of the on-sky commissioning period. Canopus was installed on the telescope the 10th of January, and nighttime commissioning started on January 
20, 2011. The first phase of the commissioning lasted five months, with five runs of 4 to 7 nights each (see Table 1). The main focus of this first period was the commissioning of LGSF and all the basic functionalities of Canopus. First "engineering-light" was obtained in April 2011. ${ }^{13}$ At this stage performance optimization was not the goal, yet the first images were already demonstrating the AO correction over an extended FoV. At the end of this commissioning period the main loops and offloads as well as the main functionality of the LGSF were operational. More details on the commissioning results out of this first period can be found in previous papers. ${ }^{6,13-15}$

After this first period of commissioning, in early June 2011, GeMS entered a planned five-month maintenance period. This shutdown was planned to perform upgrades of sub-packages development that was put on hold prior to the accelerated commissioning plan starting in January 2011. The Chilean winter yields conditions less favorable for AO observations, thus this presented a timely opportunity to fix, repair and upgrade many GeMS systems based on the experience acquired on-sky.

A second period of commissioning started in November 2011, with 7 runs of 5 to 9 nights, spread over 7 months. The objectives of this period were to demonstrate the MCAO correction, conduct science commissioning and start the integration of GeMS into the Gemini operations scheme. Wide-field corrected images were obtained during the second run in December 2011. Figure 4 is one of the first images to be acquired with GSAOI. It targets a portion of the globular cluster NGC288, the field of view on this image is $87 \times 87$ arcseconds, which is about 16 times larger that any other current AO system. This image truly illustrates the power of MCAO, namely a uniform image quality across a large field-of-fiew. Performance at that point was still limited by static residual aberrations, thus the Strehl Ratio (SR) measured in this image was only around 15\%, but with a variation of only a few percent across the whole field. We refer the reader to our companion paper ${ }^{1}$ for a detailed analysis of the performance on this image. The integration of GeMS within the observatory high-level software and telescope control and the science commissioning of GSAOI were the focus of the following runs in January and February 2012. GSAOI commissioning results are described in Carrasco et al. ${ }^{16}$ The March run was focused on finalizing GeMS functionalities and working on system stability. At the end of this run, all the loops and offloads were working and optimized, and all the interactions with the telescope were functional. Science commissioning was planned for April's run, however almost the entire run was lost due to bad weather and exceptionally poor seeing.

In June 2012, GeMS entered its second 5-month shutdown phase. This engineering period is devoted on the latest upgrades and integration required before the transition of GeMS into regular operations planned for 2013. One of the main drivers for this shutdown is also to fix the throughput of the NGSWFS. During the integration at the base facility, we uncovered an issue with the NGSWFS throughput that reduces its sensibility by approximately 2 magnitudes. A first module has been re-designed during the 2011 shutdown (see section 2.3). A second generation module will be implemented during this 2012 shutdown.

Table 1 summarizes all the commissioning nights to date. In total, 81 nights have already been allocated for GeMS commissioning. Over these nights, 14.5 were lost due to the weather. The vast majority of the weather lost was due to clouds, however, around 1.5 nights were lost due to uncharacteristically bad seeing $(>1.5$ arcsec). On the other hand, a total of $\sim 12$ nights were lost due to major technical issues. A major technical issue is considered as something that completely stops the commissioning until it is solved. The fewer major technical issues at the beginning of the commissioning can be explained by the fact that at that time we were running in a very low-level engineering mode, and commissioning per se only started in March 2011. The rise in technical issues as seen after December is linked with the beginning of the integration of GeMS with the telescope environment and the streamlining of the observations. From Decemeber on, it is interesting to note that the number of major technical issues decreased over the runs, demonstrating a general stabilization of the system. Overall, any technical issues were more frequently seen at the beginning of the runs, and usually solved in a very short time-frame by the engineering team. However this implies the need for a large engineering team either present on the summit or on-call, complicating the practical organization of the runs.

The main major technical issues that we encountered were first related to the laser and the LSE. In May 2011, one of the laser diodes failed, and in November 2011 we had temperature stabilization issues in the LSE, resulting in laser instabilities. These problems were solved in a timely fashion. A second source of major technical issues were related to the BTO and the control of its mechanisms. Major instabilites in the BTO control were found, and eventually completely fixed. A third source of major technical issue came from the real-time boards of the 


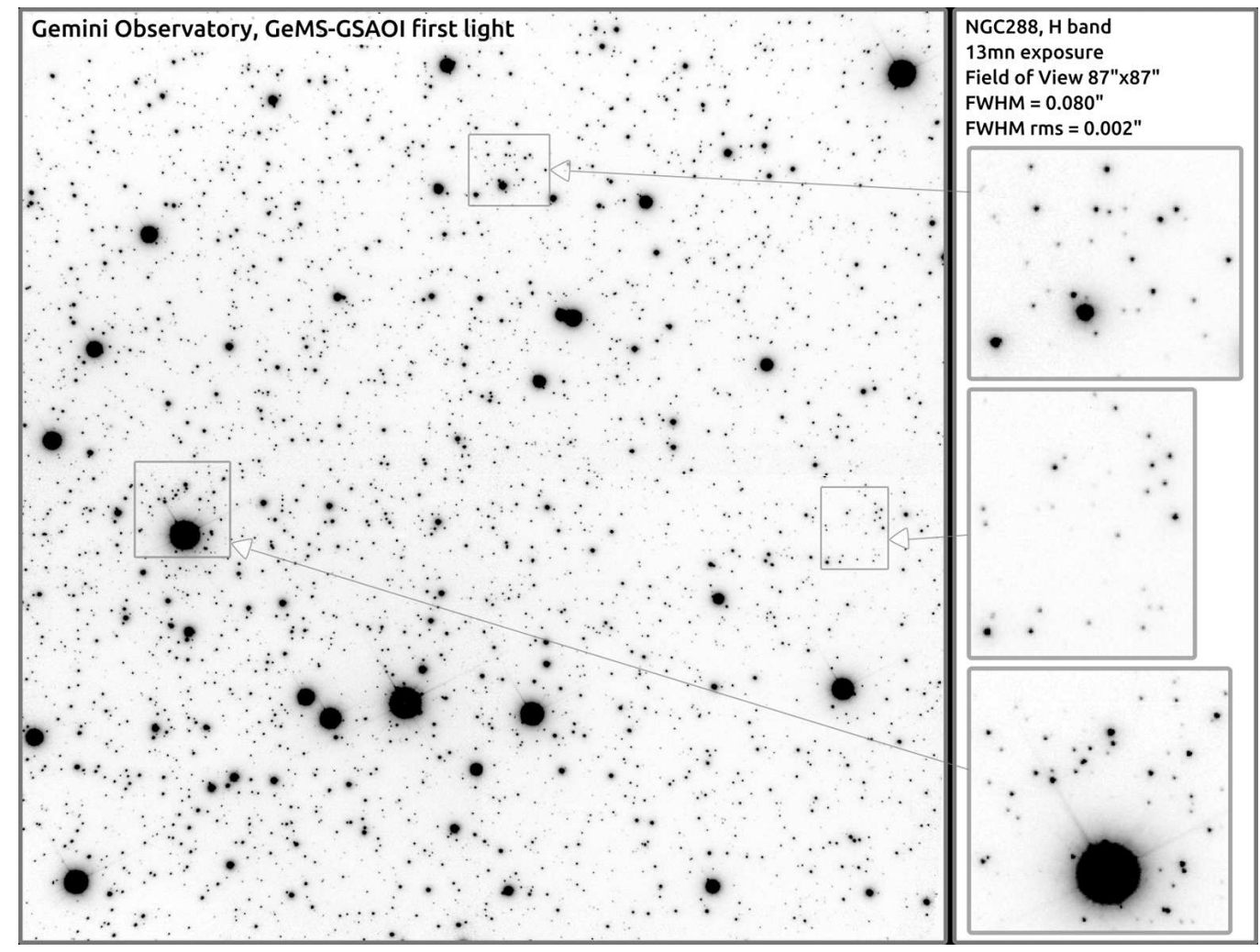

Figure 4. NGC288. The FWHM measured in this image is $80 \pm 2 \mathrm{mas}$ at 1.65 microns (H band). The field-of-view is $87 \mathrm{x}$ 87 arcseconds.

Canopus RTC. We have had episodes of of failure of these real-time processing boards - D6PC TigerSharc Board manufactured by Bittware - with the operating system that we overcame with the installation of spare boards. However, intensive and thorough diagnostic routines ran on the boards show that they are performing within specifications. More investigation on the stability of these critical boards is under investigation, and in parallel a full RTC spare is being built. The last major technical issue faced during GeMS commissioning was related to the reflective memory fiber-ring link, in charge of the time critical communication between the AO bench, GSAOI and the secondary mirror of the telescope (for Tip-Tilt offloads). In some occasions, this communication channel was broken, leading to a loss of control on the secondary mirror of the telescope. This issue will be addressed during the 2012 shutdown. Finally, and not directly related to faults, 0.5 night was lost due to a closure imposed by the Laser Clearing House (LCH) in April 2011.

After more than one year of intense work GeMS is nearly commissioned. From the engineering point of view, only few items are missing, including: control and characterization of the NGS and science ADC, control of the science beam splitter, and control and characterization of the BTO polarization. All of these should be addressed during the 2012 shutdown. From the performance side, a fine analysis and optimization of GeMS performance is still pending. Finally, from the operational point of view, more automation and operational software are needed. Most of these issues will be addressed during the 2012 shutdown.

\subsection{GeMS Overall Performance}

A detailed analysis of the performance is presented in our companion paper. ${ }^{1}$ Here we summarize the main results and expected performance.

\subsubsection{Strehl and FWHM}

Typical Strehl and FWHM obtained for different seeing conditions are summarized in Table 2. It is important to note that these are the first results derived from the given conditions. Most of the uncertainties lie in the fact 
Table 1. Summary of the GeMS commissioning nights.

\begin{tabular}{|c|c|c|c|}
\hline Date & Number of nights allocated & Nights lost to weather & Night lost to technical issues \\
\hline January 2011 & 4 & 0.4 & 0 \\
\hline February 2011 & 7 & 0 & 0.5 \\
\hline March 2011 & 7 & 0 & 1.25 \\
\hline April 2011 & 7 & 1.75 & 0.1 to 0.5 due to LCH \\
\hline May 2011 & 6 & 1.25 & 2.25 \\
\hline November 2011 & 6 & 2.2 & 0.75 \\
\hline December 2011 & 7 & 0.2 & 0.75 \\
\hline January 2012 & 6 & 1.5 & 1.0 \\
\hline February 2012 & 9 & 0.25 & 2.75 \\
\hline March 2012 & 9 & 0 & 1.2 \\
\hline April 2012 & 11 & $5.5+1.5$ & 1 \\
\hline May 2012 & 2 & 0 & 0 \\
\hline TOTAL & $\mathbf{8 1}$ nights & $\mathbf{1 4 . 5}$ nights & $\mathbf{1 2}$ nights \\
\hline
\end{tabular}

that for a same integrated seeing the final performance could be very different depending on the conjugation altitude of the main layers and their horizontal speed. As commissioning continues we are gathering more data to refine these numbers. At the same time, a full performance characterization effort is on-going, ${ }^{1}$ with focus on trying to correlate the MCAO performance with the Cn2 profiles derived from an embedded SLODAR ${ }^{17}$ (see section 6.2). It is interesting to note that for bad seeing conditions (IQ > 85, seeing > 1.0"), GeMS still provides an improvement on the image quality. Two examples are illustrated in Fig.5. The FWHM obtained on NGC4603 (Fig.5-left) are on the order of 150mas in J, 100mas in $\mathrm{H}$ and 90mas in Ks. The seeing was oscillating between 0.8 and 1 arcsec during this observation. The FHWM obtained on NGC3918 (Fig.5-right) are 120mas in Ks and $\sim 250$ mas in FeII. The seeing was less favorable during this observation, oscillating above 1 arcsec. It is expected that performance in poor seeing conditions can be improved even further pending more optimization work.

Table 2. Trypical Strehl and FWHM obtained for different natural seeing conditions.

\begin{tabular}{|c|c|c|c|c|}
\hline & J & H & K & Natural seeing \\
\hline Strehl avg. & $15 \%$ & $25 \%$ & $35 \%$ & $<0.8$ arcsec \\
FWHM avg. & $50-60 \mathrm{mas}$ & $55-70 \mathrm{mas}$ & $60-65 \mathrm{mas}$ & $<0.8$ arcsec \\
\hline \hline FWHM avg. & $150 \mathrm{mas}$ & $100 \mathrm{mas}$ & $90 \mathrm{mas}$ & $0.8-1.0$ arcsec \\
\hline FWHM avg. & $300 \mathrm{mas}$ & $250 \mathrm{mas}$ & $200 \mathrm{mas}$ & $1.0-1.2 \operatorname{arcsec}$ \\
\hline
\end{tabular}

In Fig.6 we show some examples of PSFs that have been extracted at different locations over the $85 x 85$ arcsec GSAOI field of view from NGC288 (Fig.4). The PSF uniformity over the field is extremely good, with typical variations of the FWHM by few percent only.

\subsubsection{Sky coverage}

We have estimated the sky coverage achievable by GeMS by running random pointings on the portion of the sky reachable at Gemini South. Assuming a limiting magnitude of $\mathrm{R}=17.5$ (which should be the case after the NGSWFS upgrade, see section2.3), we find that about $56 \%$ of the random pointing have three natural guide stars or more. Only 13\% have no guide star at all. The map in Fig. 7 shows how the fields are distributed in the sky. 

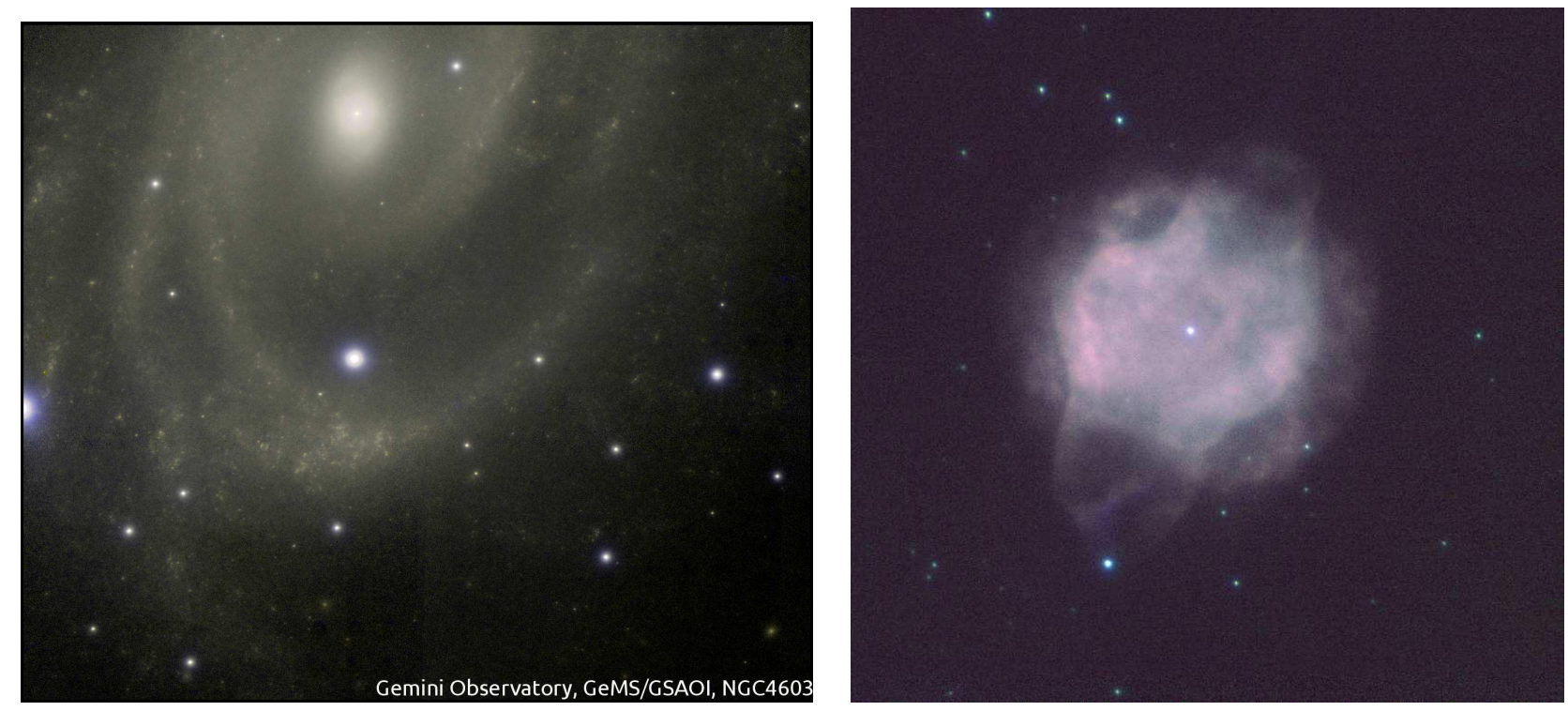

Figure 5. Left: NGC4603. FWHM measured on the image are 150mas in J, 100 in H, 90 in Ks. Seeing was between 0.8 and 1arcsec. Right: NGC3918. FWHM measured on the image are 120mas in Brg and Ks, and 250mas in FeII. The seeing was varying around 1 arcsec for this observation.
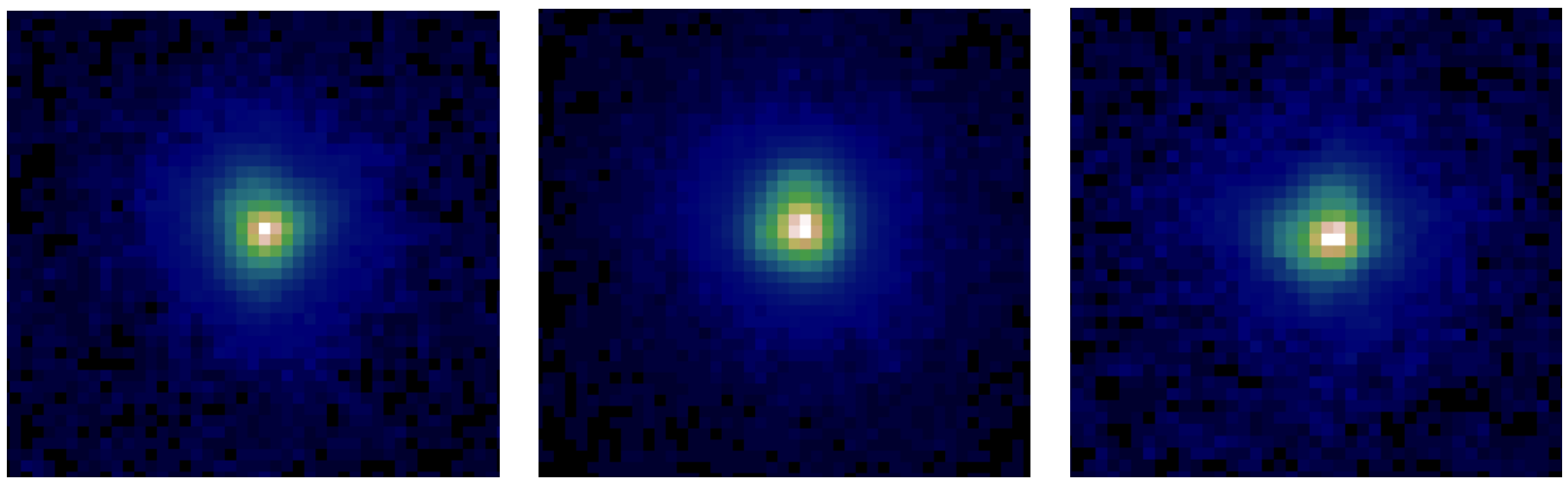

Figure 6. Example of three PSFs extracted from the $85 \times 85$ arcsec GSAOI FoV.

Black dots are fields with 3NGS, Green has no star, blue has 1 NGS, red has 2 NGS. The sky distribution plot demonstrates what could readily be expected: the highest probability to find 3TTGS is in the galactic plane, and actually extends quite far out of it. Closer to the galactic poles there is approximately equal mix, with no obvious spatial signature of points with 0 (green), 1 (blue) and 2 (red) NGS.

\subsubsection{Elevation, weather and seeing limitations}

Limitation in elevation. While the telescope elevation hard-limit is 17 degrees, the limitation for propagating the laser is 40 degrees in elevation. This limitation is imposed by the LGSWFS zoom mechanism, which cannot mechanically keep the LGS in focus at lower elevations. In addition, the LGS return flux decreases significantly at high airmass which also affects the performance.

Safety concerns with clouds. Because of safety concerns, the laser cannot be propagated in clouds that could hide any nearby aircraft from our laser spotters. The laser can be propagated in thin cirrus, however as expected the cirrus will reduce the laser brightness and affect the AO performance.

High winds. High winds entering into the dome will create wind shake and could make the Tip-Tilt loop unstable. We found that the TT loop could survive with wind on the secondary of up to $2.5-3.0 \mathrm{~m} / \mathrm{s}$. 


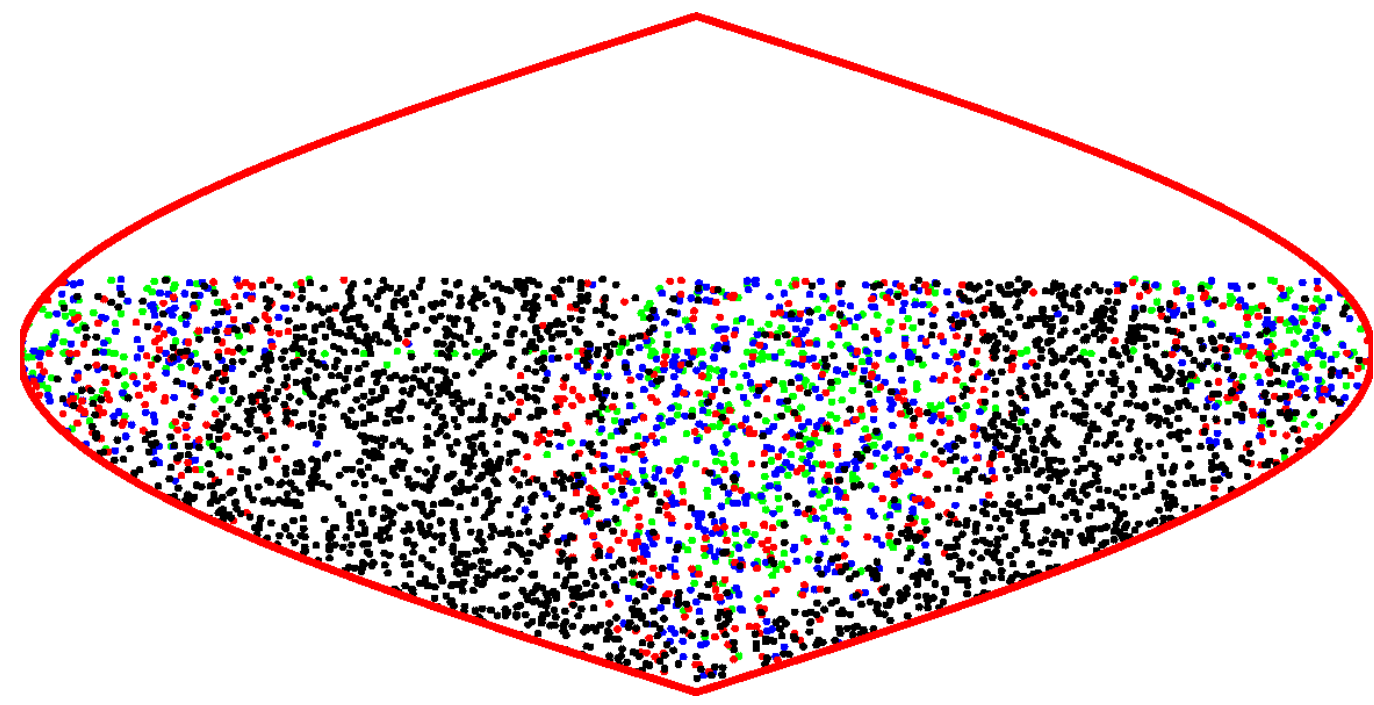

Figure 7. NGS distribution for the observable sky at Gemini South: black dots are fields with 3 NGS, red with 2, blue with 1 , and green with no natural guide star.

Transiting objects. Objects that transit near to zenith also impose a limitation. Since the telescope is on an alt-azimuth mount, the instruments, mounted at Cassegrain focus, must rotate to maintain the image orientation. This effect is especially pronounced as an object transits through zenith. As Canopus is also mounted at the Cassegrain focus, it is rotating too. However because the LGSs are launched from a fixed mirror on the back of the secondary mirror, they will be seen as rotating on sky (while the natural image remains fixed). Thus the laser constellation must also follow and de-rotate to keep the LGS spots fixed on the LGSWFS. The speed at which the laser can rotate is limited by the safe rotational speed of a mirror inside the BTO. We found that for an object transiting at an elevation of 80 degrees the laser loop would hold up to $+/-2$ minutes of the transit.

Poor seeing. Finally, for seeing worse than 1.5 arcseconds, the MCAO loops begin to lose their stability.

\section{GEMS SUB-SYSTEM PERFORMANCE}

In this section, we review the performance of each of the sub-systems of GeMS. All of these sub-systems and their interactions are presented in Fig. 8.

\subsection{Laser and BTO}

The performance of the Laser and BTO are presented in a companion paper ${ }^{8}$.

\subsection{LGSWFS and high-order loop}

LGSWFS assembly. The LGS assembly contains 8 stepper mechanisms (two zoom lenses and six magnificators) used to accommodate for the changes in range of the LGSs (change in telescope elevation or changes in the $\mathrm{Na}$ layer altitude), as well as to compensate for flexure and temperature variations present at the ground level. The current range accessible with the LGSWFS is from $87.5 \mathrm{~km}$ to $140 \mathrm{~km}$, corresponding to an elevation range of 90 to 40 degrees respectively. A Look-Up Table (LUT) has been made by moving the telescope in fixed elevations and rotator positions. The parameters that need to be controlled are: (i) the DM0 to each LGS WFS registration, (ii) the WFS magnification and (iii) the focus phase errors. The zero-points of these LUT are updated at the beginning of each night for the temperature. This is done by an automatic procedure that optimizes the registration for each $\mathrm{WFS}^{18}$. Direct on-sky measurements have shown that the registration is better than $10 \%$ of a WFS sub-aperture ${ }^{1}$.

The 5 LGSWFS are using E2V CCD39-AIMO a very low dark current type of detectors. The Read Out Noise (RON) of these CCDs has proven to be very good at approximately 4 electrons. The CCD noise is monitored 


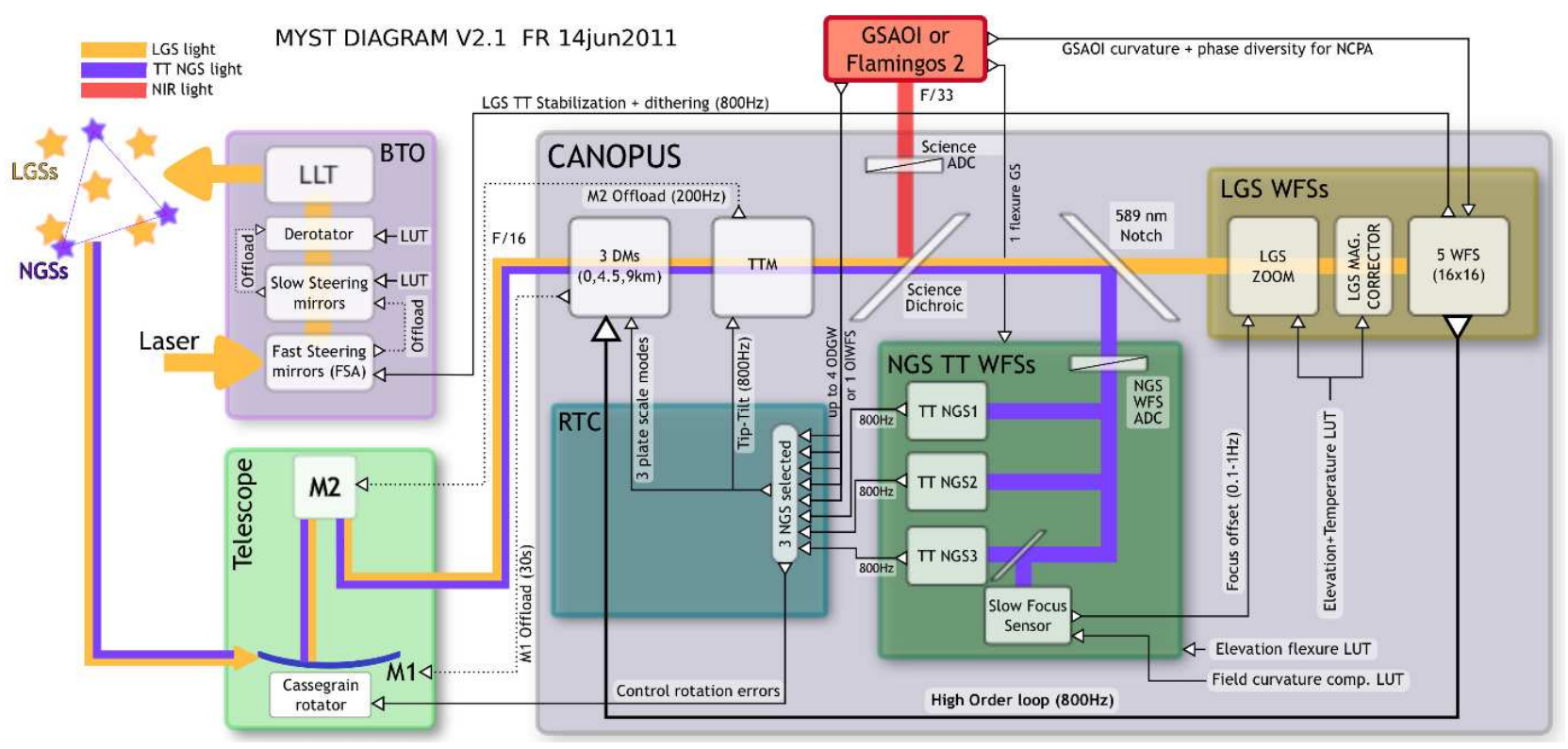

Figure 8. Overview of all GeMS sub-sytems, loops and offloads.

daily and during the night, as this parameter is particularly important with a low sodium return ${ }^{19}$. These E2V CCDs suffer from Pixel Edge Diffusion (PED) producing an extra equivalent gaussian kernel of $\sim 1$ arcsec. Different waveform and bias values have been tested to reduce this effect, but no significant improvements have been achieved so far. We are planning to allocate more time to work on this very subtle problem once other higher priority tasks are completed.

The main issue we had with the LGSWFS assembly was related to a field stop vignetting. In January 2011, one of the magnificators was giving positioning errors and had to be serviced and then reinstalled, resulting in a misalignment of the LGSWFS field stop, reducing the clear field by \pm 0.3 arcsec. The vignetting was only detected in April, when the seeing conditions were worse than usual, and shadows on the sub-apertures could be seen. A direct impact of this vignetting on the performance was to create large instabilities in the Fast Steering Array (FSA) and high-order loop. Affected subapertures would also see errors in their centroid gains, leading to Non Common Path Aberration (NCPA) leaks into the science path.

Limiting magnitude. We generally work at about 140 to $160 \mathrm{ph} /$ subaperture/frame or between 35 and $40 \mathrm{ph} / \mathrm{pixel} /$ frame, as the LGSWFS is working with $2 \mathrm{x} 2$ pixels/subaperture. To maintain this flux level, the sampling frequency is adjusted: during the low sodium season (December and January) a typical guiderate is about $200 \mathrm{~Hz}$, while during high sodium season (April and May) the guiderate was between 400-800 Hz.

Deformable Mirrors. GeMS uses three piezo-stack DMs manufactured by CILAS. However, following the failure of 19 actuators (out of 240) in just a short period of time, the DM0 (conjugated at the ground layer) was removed from the optics bench and replaced by the DM conjugated at $4.5 \mathrm{~km}$. This DM and the $3 \mathrm{rd}$ one conjugated at $9 \mathrm{Km}$ were also made by CILAS but under another manufacturing process. During the first commissioning period, the rate of failure for DM0 was about 1 more dead actuator every 10 days. Currently we are working with only $2 \mathrm{DMs}$ conjugated to 0 and $9 \mathrm{~km}$ above the site, reducing the total number of actuators originally planned by more than a factor of two. By means of simulation, we have estimated the impact on the performance to be between $5 \%$ and $15 \%$, depending on the turbulence profile. Fortunately a new DM has been ordered and should be received by 2013 .

\subsection{NGSWFS, and focus loop}

NGSWFS probe design. Physically mounting two of the Canopus Tip-Tilt star probes (C1 and C2) onto the third probe mechanical assembly (C3) minimizes both the differential flexure between the probes and any 
resulting changes in the plate scale on the image. During the acquisition, all probes are moved independently to find their respective star (see astrometric mode below). However, once the probes are in position, $\mathrm{C} 1$ and $\mathrm{C} 2$ are locked to C3, fixing the relative distance between the 3 probes. The C3 probe can move to track the Tip-Tilt star constellation through telescope offsets, to implement any flexure compensation model or for any other general purpose. Probes $\mathrm{C} 1$ and $\mathrm{C} 2$ are then moving by the exact same amount, conserving the image plate scale and allowing for astrometry measurements. The 3 probes lie in different planes to avoid collisions between them. $\mathrm{C} 1$ can vignette $\mathrm{C} 2$, which can vignette $\mathrm{C} 3$. The vignetting area is however small, the width of the arm is $10 \operatorname{arcsec}$. If necessary, the field can be rotated to avoid any vignetting.

Limiting magnitude. The main limitation of the NGSWFS has always been its throughput. This is a known issue since Canopus started its integration in the laboratory. Due to design errors and alignment issues, the original setup suffered from more than a 2 magnitude sensitivity loss. Most of the light loss was happening at the injection of the light into the fiber, and the coupling between the fiber and the APDs (see Fig.3). New APD modules were purchased and installed, providing a better coupling and a gain of $\sim 1.5$ in flux. A new fiber injection module has also been designed and implemented for one of the probes $(\mathrm{C} 1)$. We found that the transmission from the entrance of the probe to the output of the fiber went from $50 \%$ to $66 \%$. The theoretical transmission is about $77 \%$, so this new design could be improved even further. In Table 3 we summarize the flux measured on-sky, for a $\mathrm{R}=15 \mathrm{mag}$ star with the different upgrades that have been made. As a reference, we compare the numbers with those obtained by Altair, as it uses a TT WFS with a similar APD quad-cell concept (although different in its implementation, as Altair does not use fibers but a STRAP unit that feed the light directly from a focal plane lenslet array to the APDs themselves). Even though the upgrades made during the past months allowed for an improvement of the transmission by a factor of $\sim 2$, the current throughput is still a factor of $\sim 2$ lower compared to Altair. During commissioning, we have empirically determined that $\sim 50$ photons per frame are required for the Tip-Tilt loop, which when running at $100 \mathrm{~Hz}$ has a limiting magnitude of $\mathrm{R}=16.5$.

Table 3. NGSWFS sensitivity: number of photons detected (total) for a $R=15 \mathrm{mag}$ star.

\begin{tabular}{|c|c|c|c|}
\hline Original design & With new APDs & With new fiber injection module & Altair \\
\hline $10 \mathrm{ph} / \mathrm{ms}$ & $15 \mathrm{ph} / \mathrm{ms}$ & $20 \mathrm{ph} / \mathrm{ms}$ & $46 \mathrm{ph} / \mathrm{ms}$ \\
\hline
\end{tabular}

Optical distortions. During commissioning, we found that the NGSWFS was suffering from large optical field distortions. These distortions are seen on the science images as well and they can be as large as 1 arcsec. As the position of the NGS on the NGSWFS focal plane fixes the plate scale, it will be affected when the telescope pointing is different. In other words, the relative distance between the NGS changes when the whole constellation is translated in the NGSWFS focal plane affecting the plate scale on the science image. A detailed analysis of this effect and possible mitigations are presented in our companion paper ${ }^{1}$.

Probe mapping. At the beginning of an observation, the coordinates of the three NGS loaded from the science program must be converted into coordinates in the focal plane, and motor positions for the three NGS probes. This is done through a linear mapping of the different coordinate systems. For the third NGS probe (C3) the transformation is fairly straightforward consisting in a $2 \times 2$ matrix plus a constant term. The formula describing the probe coordinates for $\mathrm{C} 1$ and $\mathrm{C} 2$ are more complicated, since they rely on the position for $\mathrm{C} 3$. Hence, a first transformation that converts the $\mathrm{C} 1$ and $\mathrm{C} 2$ probe coordinates in the reference frame of $\mathrm{C} 3$ is needed. In terms of solving the transformation equations, this design and set of models led us to solve for the $\mathrm{C} 3$ transformations prior to the $\mathrm{C} 1$ and $\mathrm{C} 2$ transformations. This was done because without a reliable $\mathrm{C} 3$ transformation, it is very difficult to determine whether an error in the $\mathrm{C} 1$ model is due to coefficients unique to $\mathrm{C} 1$ or due to those in the C3 model.

The procedure for determining the $\mathrm{C} 3$ model coefficients was to acquire a star near the center of the field and offset the telescope by about 10 arcseconds. The star was then reacquired and probe positions were recorded. This was done in 2 axes producing a crude model for probe offset and scale. This initial model was refined with larger offsets, about 60 arcseconds, in locations around the edge of the patrol field. Since each telescope offset provides 2 constraints on the model and C3 has 6 free parameters, 10 telescope offsets were sufficient (20 constraints) to provide a reliable model for $\mathrm{C} 3$. After the $\mathrm{C} 3$ model was determined, the same procedure was 
repeated for $\mathrm{C} 1$ and $\mathrm{C} 2$ models. At this point, stars were usually within about 1 arcsecond of the expected probe position.

This entire procedure took about 1 night of telescope time, although in reality is was split over many nights interspersed with other instrument commissioning activities. Once this probe mapping was accomplished, additional refinement was useful. Our goal, to have the root-mean-square (RMS) deviation of the probe target residuals to be on the order of 0.5 arcsecs, was chosen as the RMS needed to allow most stars to be acquired in the $\sim 1.5$ arcsec field of view of the Tip-Tilt sensors. To achieve this accuracy, we used a large number of Tip-Tilt star constellations on a variety of targets, elevations, and Cassegrain rotator positions. This additional refinement was done in parallel with other commissioning activities, adding only a few minutes of extra time to acquisitions. A total of 70 stars were used to refine the model. Figure 9 shows the residuals measured with the final model. The RMS is about $0.3 \mathrm{~mm}$, which corresponds to 0.5 arcseconds given that $1 \mathrm{~mm}$ corresponds to 1.6 arcseconds in the focal plane. More data has been gathered, however it is difficult to improve the models significantly as the residual error is due to a combination of catalog accuracy, non-compensated flexure, and optical distortions in the NGSWFS focal plane, which cannot be taken into account by linear models.

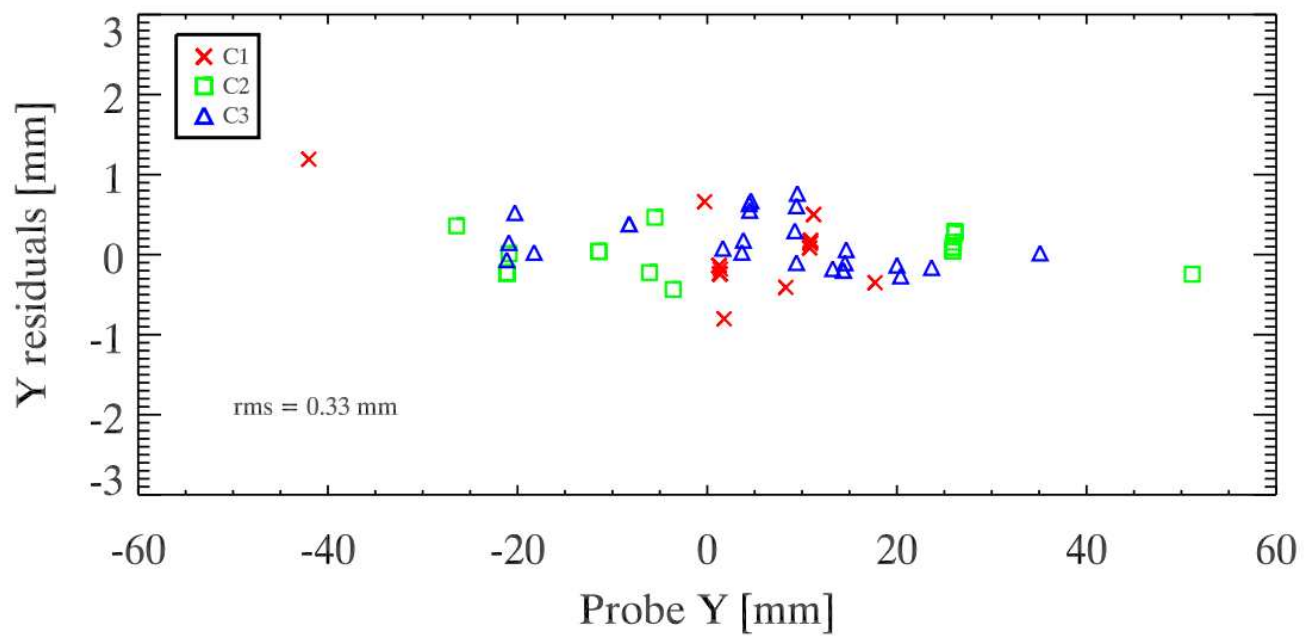

Figure 9. Residuals errors in Canopus probe mapping, obtained with the final model. The RMS is about $0.3 \mathrm{~mm}$, which corresponds to 0.5 arcseconds. No obvious bias can be seen over the field or for the different probes. We only display the error for the $\mathrm{Y}$ axis, similar errors are measured for the $\mathrm{X}$-axis.

Astrometric mode. The differential Tip-Tilt errors between the three probes are used to control the plate scale mode (also called Tilt-Anisoplanatic or TA loop). The plate scale errors are compensated by applying quadratic modes on both DM0 and DM9. Dynamical range for DM0 is not too much of an issue, as its static shape is offloaded to the primary mirror of telescope. However, there is no offloading possibilities for DM9. Therefore, the position of the probes in front of their respective GS must be optimized. This is what we call the astrometric mode. In practice, once the stars are close enough to their respective WFS, the Tip-Tilt errors are averaged over a 10s period of time, and each of the NGS guide probes is moved in order to minimize this error. Once the residual Tip-Tilt errors are below a given threshold depending on the required astrometry accuracy the probes are set to their position.

Guiding options. Different guiding options have been tested during the commissioning, including guiding on non-sideral objects, extended objects and stars embedded in high background environment. We have successfully guided using three of Saturn's moons with the telescope centered on the planet. It is also possible to lock the telescope on a non-sideral target, and guide using background stars. A combination of sidereal and non-sidereal guiding, while possible, has not been tested. Guiding on diffuse galaxy cores reduced the performance and stability of the Tip-Tilt loop. Finally, stars embedded in high-background have also been used successfully, for instance during observations in the Orion Nebulae. 
Slow Focus Sensor (SFS). As we are using the LGS to compensate for atmospheric focus, we cannot disentangle any changes in the sodium layer altitude from real atmospheric focus changes. In other words, if the altitude of the LGS varies (by a few hundreds of meters) we will interpret this as focus, and the DMs will compensate for it. The focus will change in the science path as well. To cope with this effect, we need a "truth focus sensor" which monitors the true focus on an NGS. This is done by the SFS: a 2x2 Shack-Hartmann sensor using $30 \%$ of the light from NGS probe 3. The error in focus as calculated by the SFS is sent to the LGSWFS zoom to track the best focus position. We have estimated that 50nm of focus (that's a loss of $4 \%$ of $\mathrm{SR}$ in $\mathrm{H}$ ) corresponds to a centroiding accuracy of 0.1 pixels. The centroid of the spots is determined based on a thresholded center of gravity. Correlation and Gaussian fitting has also been implemented, but leads to similar results. The focus adjustments have been implemented based on the following equation:

$$
\text { Focus }=F_{z e r o}+F_{\text {off }}+F_{\text {integ }} \text { where } F_{\text {integ }}=F_{\text {integ }}-g *\left(F_{\text {off }}-F_{\text {ref }}-F_{\text {mes }}\right)
$$

where $F_{\text {off }}$ is a term that allows to adjust the focus both in close loop and open loop, $F_{\text {ref }}$ allows to adjust the focus in close loop, $F_{m e s}$ is the actual focus measurement, $g$ is the gain of the close loop and $F_{z e r o}$ is an offset that can be used to adjust the focus for different instruments, or different filters. Useful focus measurements can be obtained with a 1 s exposure time for NGS with $\mathrm{R}<13.0$. To keep associated errors minimal (1\% SR loss in H-band) the focus values should update at a rate no greater than once every 30s. As a result, our current limiting magnitude is $\mathrm{R}=16.7$.

During commissioning, we found that the focus varies within the NGSWFS focal plane. This is a potential issue because the reference focus will change depending on the position of $\mathrm{C} 3$ in the field. If this cannot be fixed by optimizing the optical alignment, a LUT for the focus reference across the field should be implemented.

NGSWFS upgrades. To cope with the current throughput limitation described above, we are working on a second generation fiber injection module. The main difficulty encountered in the first prototype was in the alignment of the assembly pyramid + fibers. In fact, the tolerance in the pyramid fabrication is such that the position of each fiber should be optimized independently. This was not possible in the current setup. The new prototype provides the required degree of freedom to optimize the transmission at the entry of each fiber. This should enhance the transmission by $\sim 25 \%$. The remaining loss in transmission has been identified as coming from the LGS beam splitter (BS2 in Fig.2). Replacing this optic should improve the limiting magnitude to a level comparable to Altair $(\mathrm{R}=17.5 \mathrm{mag}$ at $100 \mathrm{~Hz})$.

In parallel to this activity, we are studying an alternative design based on the EMCDD ${ }^{20}$ or sCMOS ${ }^{21}$ technology. The idea is to replace the whole NGSWFS assembly by a detector in the focal plane, and measure the centroids of the stars to derive the Tip-Tilt measurements.

The new sCMOS array would potentially open the possibility of having multi-window in a same chip. These small windows can be read at a rate of up to $800 \mathrm{~Hz}$, with a very low noise $(<1 \mathrm{e}-)$. The current size of the sCMOS array (more than $1 \mathrm{k}^{2}$ ) is compatible with the requirement of covering the 2 arcmin patrol field, with Shanon sampled stars. Considering that the NGSWFS benefits from the MCAO correction, the best FHWM of the stars (in the visible) would be around 0.4 arcsec. Using $0.2 \operatorname{arcsec}$ pixels, an array of $600 \mathrm{x} 600$ would cover the required field.

In the EMCCD option, and because multi-windowing is not possible, a mosaic of four arrays should be considered. Then one NGS per array could be used for the guiding. This solution would be very close to the ODGW capability of GSAOI. ${ }^{12}$ However, this configuration is less optimal as it restricts the number of possible constellations.

Replacing the NGSWFS assembly by a focal plane array should improve the global transmission: we have estimated the gain to be of the order of one magnitude, fulfilling the $\mathrm{R}=18.5$ limiting magnitude requirement. Moreover, this will also significantly simplify the acquisition procedure as one can take advantage of the full frame image. Finally, the compensation of the optical field distortion will also be simplified. The main drawback of this solution is that an alternative sensor providing the slow focus variations must be designed. Two options are currently considered: the first uses a peripheral WFS of the telescope (as it is currently done for the Altair "super seeing" mode ${ }^{22}$ ), the second one would be to use the On-Instrument WFS (OIWFS) or directly the focal plane images provided by the ODGW in the GSAOI case (see section6.3 for more details). 


\subsection{Flexure loop and fast ODGW guiding}

Flexure loop. The flexure loop uses the signal coming from an On-Instrument WFS to compensate for potential differential flexure between the AO bench and the instrument. The flexure signal is used as soon as the Tip-Tilt loop is closed by moving the three Tip-Tilt WFS probes all together. As the Tip-Tilt loop is closed on the signal coming from these probes, moving the probes moves the Tip-Tilt mirror accordingly, and results in image motion at the science detector level. First results obtained on-sky have shown that the differential flexures between the $\mathrm{AO}$ bench and (for instance) GSAOI are small. We measured image motion on the order of $\sim 0.2$ arcsec on-sky for 30 degree elevation steps.

Fast ODGW guiding. As described in the introduction, in the case of GSAOI, a second mode of operations is offered by using the ODGW to provide the fast Tip-Tilt guiding. The ODGW technology is described in Young et al. ${ }^{12}$. This mode has not been commissioned yet, and only first tests using the calibration sources were conducted. The Tip-Tilt loop has been successfully closed with the ODGW. Figure 10-left shows an image of Canopus calibration sources acquired with the four ODGWs, and Fig.10-right an Error Transfer Function (ETF) derived for the ODGW. It is measured as the ratio between the Power Spectral Density (PSD) of the Tip-Tilt error signal measured in close loop and in open loop, while working on noise-only conditions. The bandwidth achieved is around $20 \mathrm{~Hz}$, which is in good agreement with the performance obtained when using the Canopus visible Tip-Tilt WFS. These first results are demonstrating the basic functionality of the ODGW.
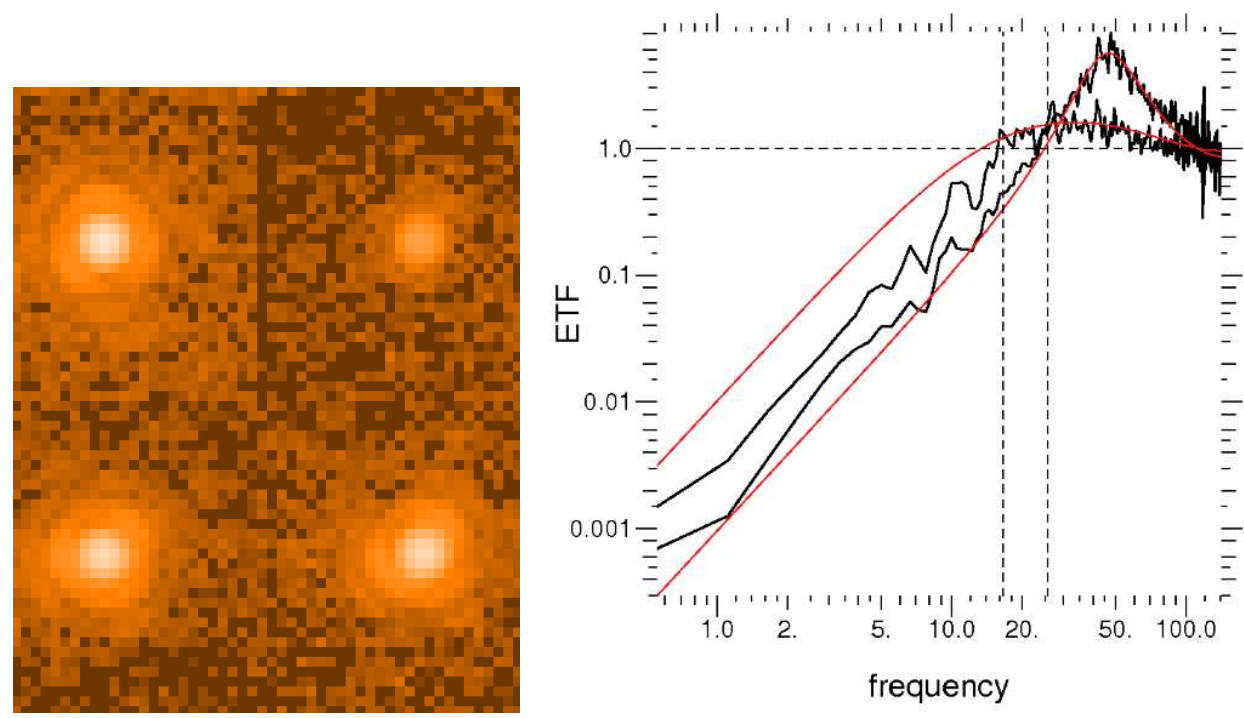

Figure 10. . Left: Four ODGW image of the Canopus calibration sources in K-band. Right: ODGW error transfer function (black=measured, red smooth=theoretical) obtained for the X-axis. Frame rate is $280 \mathrm{~Hz}$, for gains of 0.5 and 1.0 .

Limiting magnitude. The requirement for the fast ODGW guiding is to provide a centroid measurement accuracy of 0.1 pixels. Using the measured zero points of GSAOI, ${ }^{16}$ and simple analytical formulae for the computation of the centroid errors $\left(\mathrm{see}^{23,24}\right)$, one can determine the theoretical limiting magnitude. First results on-sky show that for a $\mathrm{K}=10.3 \mathrm{mag}$ star with $10 \mathrm{~ms}$ exposure time (frame rate $=7 \mathrm{~Hz}$ ) a Signal-to-Noise Ratio (SNR, defined as the maximum divided by the RMS of the background) of approximately 150 can be achieved. This SNR reduces to 2.5 when the exposure time goes down to $1.6 \mathrm{~ms}$, which is approximately the limit for fast guiding use, hence defining the limiting magnitude.

\subsection{ADCs and Science beam splitter}

ADCs. Canopus has two Atmospheric Dispersion Compensators (ADCs). One is located on the science path, and can be inserted or removed from the path, the other one is in the NGSWFS path, and is always in. The 
ADCs have not been commissioned yet. However, characterization of the NGS ADC with the Canopus calibration sources has revealed three potential issues when the angle and power of this ADC change. First, depending on the ADC setup, the focus term will change. As the SFS is part of one of the NGSWFS probe, a LUT to take this effect into account must be implemented. Second, the ADC is creating pupil wandering on the SFS, leading to biased focus values. Combining these two effects with the focus variations across the NGSWFS field, the implementation of a LUT appears to be quite complex. The third and most serious issue is that depending on the ADC setup the plate scale in the NGSWFS field will change. In other words, when the ADC rotates, the relative positions between the NGS are changing. The amount of differential motions measured were on the order of 50 to 100mas, almost two orders of magnitude higher than the astrometry requirement. To cope with these issues, the current strategy for the NGS ADC will be to set it at the beginning of an observation based on the average telescope elevation, and then keep it fixed. In the worst case of an observation spanning from Zenith to 40degree of elevation, the maximum relative elongation due to atmospheric dispersion is \pm 0.3 for optical wavelength ( 550 to $850 \mathrm{~nm}$ ). The impact on the SNR is then small compared to the complexity of having this ADC in follow mode.

Science beam splitter. A set of two dichroics is also available, with cut-off frequency of 0.85 um and 1.0 um. To date, we have only been using the bluest one $(0.85 \mathrm{um})$ allowing for $\mathrm{z}$-band observations. The final commissioning of this mechanism is currently on-going.

\section{FROM COMMISSIONING TO OPERATIONS}

GeMS is a facility instrument, thus it must be integrated into the observatory software and control system. Despite its complexity, it is planned for GeMS to be operable by a crew of only two people: the telescope operator will manage all of the AO systems, while the laser operator will be in charge of the Laser and BTO. The instruments will still be operated by an astronomer. While most of the difficult commissioning work has been completed, the path from a demonstration system to a fully-functional facility instrument is non-trivial. New tools must be developed to bridge the knowledge gap when handing over such a complicated system from a large engineering team to normal operations.

\subsection{GeMS SMART tools}

Most of the complexity of GeMS resides in the control and optimization of all the loops. There are as many as 20 loops and offloads that must be closed, monitored, and controlled. Further complicating the process, most of these loops are interacting together. Because managing all of these loops is out of the scope of the telescope operator, we have developed a layer of software called GeMS SMART tools. ${ }^{25}$ These SMART tools simplify the interaction with the AO system and integrate automation in the operation flow, assisting the operator both in the acquisition procedure and during the observation. When the operator wants to close the high-order loop, a set of conditions and commands are first executed by the SMART tools. For example, the flux is checked, low level loops are closed, default matrices are loaded, and gains and sampling frequencies are optimized. Once the high-order loops are closed, the SMART tools continue to monitor the status and performance of all loops and can even make ("smart") decisions and take actions according to the external conditions to maintain the performance. Figure 11 shows a screenshot of the user interface to the SMART tools. In practice the SMART tools have proven to be very useful for the transition into operations.

\subsection{Acquisition procedure and overheads}

One of the main tasks carried out during the second commissioning period was to define and optimize the acquisition procedures. Our most recent procedure iteration consists of seven steps, each requiring human intervention either from the telescope or laser operator. While this current procedure is already much more efficient than initial acquisition procedures, more automation is under development to reduce the overheads even further. Below, we describe each of the steps required for an entire GeMS acquisition in $3+1$ mode, as fast guiding with the ODGW has not yet been commissioned. This procedure is from telescope slew to the beginning of the science exposure. The actual science observation preparation is described in Carrasco et al., $\left({ }^{16}\right)$. Table 4 summarizes these steps and shows associated overheads. 


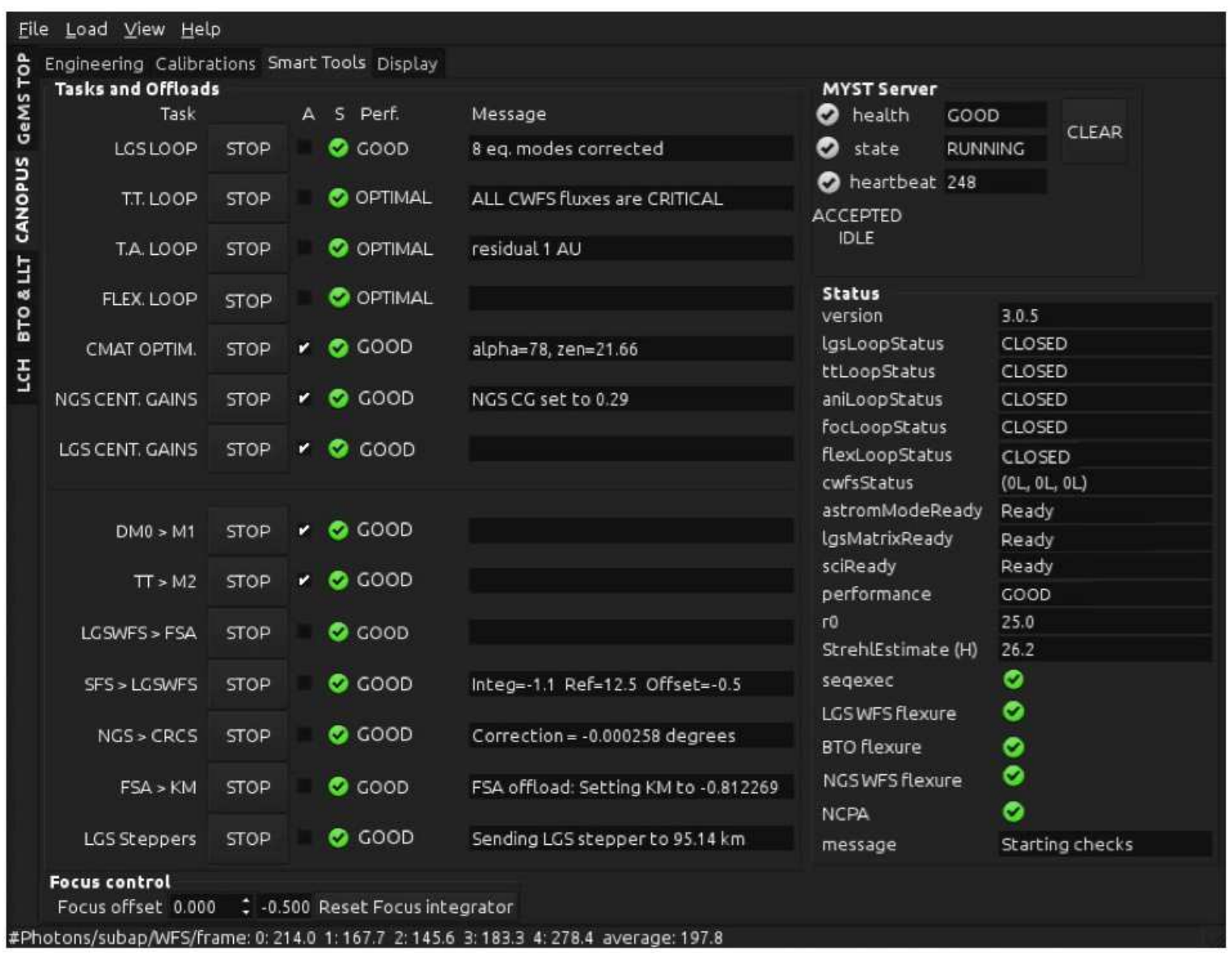

Figure 11. Screenshot of the Myst SMART tools GUI.

1. Telescope slew and mechanisms in follow. When the telescope operator slews to a new GeMS target the Myst SMART tools also receive the slew command. The SMART tools then set all mechanism offset positions to zero and, based on the telescope position and temperature, puts all mechanisms into follow according to their LUT (or with probe mapping as is the case of the NGSWFS). Once the telescope is in position, the NGS and LGS alignment are done simultaneously by the telescope and laser operator maximizing on-sky efficiency. In most cases during a slew, the GeMS mechanisms reset and reach position before the telescope, thus the overhead on this step usually comes from the telescope-side.

2A. Laser alignement. With the telescope now in position, the laser operator begins alignment of the laser inside the BTO only, at low power. This alignment consists of a handful of steps, which in total take between 3 to 6 minutes to complete.

First, the laser must be aligned up to the LLT. For safety reasons, this alignment is executed at a very low laser power of only a few $\mathrm{mW}$. Using the video signal coming from the Pre-Alignment Cameras (PACs) the laser operator can accurately center the beam on each of the BTO mirrors leading up to the LLT. Once this critical alignment has been completed, the laser operator requests permission from the telescope operator to propagate onto sky.

The telescope operator then checks for any beam collisions that might impact the science of other telescopes, and requests permission from the laser spotter stationed outside monitoring for aircraft ${ }^{8}$. With the permission to propagate, the laser operator increases the laser to medium power $(2-5 \mathrm{~W})$ and then checks the alignment of the laser beams on the LLT. Once the beams are properly centered on the LLT, the laser operator increases to maximum power and using the Rayleigh light pattern, starts the alignment of the laser constellation on the LGSWFS. A simple geometric model of the Rayleigh backscattering has been implemented and a minimization 
routine provides the geometric solution consisting of several parameters, of which the global de-pointing is the most important. This global de-pointing is applied to the BTO pointing and centering mirrors (PM/CM) to center the LGS constellation. With the constellation aligned, the laser operator closes the FSA loop keeping the constellation fixed on the LGSWFS. Finally, when everything has stabilized, the laser operator gives the green light to the telescope operator to close the high-order loop.

2B. NGS alignement. To maximize on-sky efficiency, the telescope operator starts the NGS acquisition in parallel with the laser alignment. Depending on the field and brightness of the NGS, this can take between 2-10 minutes (a crowded field, faint or diffuse NGS targets are more difficult setups). As the Gemini South telescope pointing accuracy from slew-to-slew is on average 10 arcseconds, it is necessary for the telescope pointing first to be corrected. This is a standard procedure for any telescope slew, but all the more important for GeMS acquisitions as the NGSWFS probes have only a 1.5 arcsecond FoV. To do this, the telescope coordinates are swapped from the science object to those of the C3 coordinates thereby putting the C3 star on-axis. Next, the wide-field Acquisition Camera (AC) is used (1x1' when used with Canopus) and the pointing adjusted to put the C3 target onto a pre-determined telescope hot spot. With the pointing now corrected, the telescope is reverted to the science object position and the Canopus probes, using the probe mapping model will move into position, ensuring that the star corresponding to $\mathrm{C} 3$ will be properly centered on the probe. Next, the Tip-Tilt loop is closed with C3 only, while C1 and C2 APDs are readout to check the counts. Assuming the NGS catalog coordinates are all correct, probes $\mathrm{C} 1$ and $\mathrm{C} 2$ will likely be very close to their NGS targets, and flux should be seen on the APDs. The telescope operator can then start the Myst astrometric mode, which automatically adjusts the probe positions in a more accurate manner. Once the remaining two probes are centered, the Tip-Tilt loop can be closed on all three probes. If one of the stars does not appear on the APD (e.g. because of catalog errors), the telescope operator can start an automated spiral search for the star. In the event that a star still does not show up, it is possible to swap the telescope coordinates with that guide star to look for it in the AC (as was done first with C3). If necessary, the AC images can also be compared with finder charts, helping the operator identify the correct guidestars. These additional steps require the C3 offload to Tip-Tilt to be stopped, and add additional acquisition overhead. In practice, bright guidestars in relatively sparse fields require the least amount of overhead.

3. High-order loop and dependencies. With the laser FSA offloading and the NGS Tip-Tilt loop closed, the telescope operator can now close the high-oder loop. While the command to close the loop is sent from the Telescope Control System (TCS), it is the Myst SMART tools that actually handles the proper sequencing. Default matrices and gains are automatically loaded and frame rate is optimized based on the NGS and LGS flux. Offload of the static DM0 shape to M1 is also started based on the loop performance. This entire process happens in 1-2 minutes and is monitored by the telescope operator for stability.

4. Focus loop. With the high-order laser and NGS loops closed, the telescope operator can now focus on the SFS loop. Depending on the C3 target brightness (SFS receives $30 \%$ of the C3 light), this process can take 2 to 5 minutes. First, the telescope operator starts the readout of the SFS camera with his best guess for integration time (based on a table), adjusting to optimize the SNR on all four spots. In the case of a very faint GS, the integration time can be as long as 5 minutes, explaining the large overhead for this step. When the SNR is sufficient, the telescope operator closes the SFS loop, which applies a focus term to the DM0 that is immediately offloaded to the secondary mirror of the telescope (M2). The telescope operator can see real-time the trend of the M2 focus, visually showing when the focus has converged.

5. Rotator and Tilt-Anisoplanatic (TA) loops. At this point the telescope operator can close the Rotator and Tilt-Anisoplanatic (TA) loops. The rotator loop compensates for any tracking errors of the telescope rotator, while the TA loop compensates for any differential Tip-Tilt errors between the three NGS probes, thereby fixing the plate scale. Information about this loop performance is also provided by the SMART tools GUI.

6. Flexure loop. The final step of the acquisition procedure is to close the flexure loop. This can take from 2-5 minutes. First, the telescope operator reads out the ODGW that will be used for flexure compensation. Exposure times for the ODGW are pre-defined based on flexure star magnitude and filter used by GSAOI, and can be adjusted as needed. The ODGW position is determined in a similar manner as the NGS probe mapping, and described in further detail in a separate paper ${ }^{16}$. The size of the ODGW is first set to the largest value $(128 \times 128 \sim 2.5$ arcseconds on-sky). If the star does not appear on the ODGW, a full-frame image can be taken 
with GSAOI to check its position with respect to the guide window, however this adds overhead. Once the star is properly centered on the ODGW (by re-defining the ODGW X,Y detector position, not the telescope position since we are at this point fully closed loop with the LGS and NGS T/T stars) the size of the ODGW window is reduced to $16 \times 16(\sim 0.3 ")$ and the flexure loop is closed.

7. Science ready! Finally, everything is ready for science! The telescope operator will give a final check on all the loops and offloads, and give the green light to the astronomer to start the science sequence.

Table 4 . Summary of the acquisition procedure and associated overheads.

\begin{tabular}{|c|c|c|}
\hline Step & Description & Typical overhead \\
\hline 1 & Telescope slew and mechanisms in follow & 1 to $2 \mathrm{~min}$ \\
\hline $2 \mathrm{~A}$ & Laser alignment & 3 to $6 \mathrm{~min}$ \\
\hline $2 \mathrm{~B}$ & NGS alignment and Tip-Tilt loop & 5 to $10 \mathrm{~min}$ \\
\hline 3 & High-order loop & 1 to $2 \mathrm{~min}$ \\
\hline 4 & Focus loop & 2 to $5 \mathrm{~min}$ \\
\hline 5 & Rotator and TA loops & 1 to $4 \mathrm{~min}$ \\
\hline 6 & Flexure loop & 2 to $5 \mathrm{~min}$ \\
\hline 7 & Science ready & $1 \mathrm{~min}$ \\
\hline Total & & 16 to $35 \mathrm{~min}$ \\
\hline
\end{tabular}

As seen in Table 4, the large range in setup overheads can be almost entirely attributed to the NGS acquisition. For bright NGS without catalog errors, the full GeMS acquisition has been as fast as 10 minutes. For faint NGS, or more complex objects (high background or a crowded field) the acquisition procedure can be $20+$ minutes. We have also experienced several occurrences where the NGS brightness as listed in the catalog were incorrect by several magnitudes thus a better NGS constellation needed to be chosen on the fly, greatly increasing the setup time. Because of this we are considering the possibility of pre-imaging GeMS fields. While this would add overheads to the program, pre-imaging would be fast, no more than 5 minutes for each field (accounting for the telescope slew and image with the $\mathrm{AC}$ ), and would not require good conditions.

\subsection{Dither-Filter-Sky sequence}

A science observation sequence can include telescope offsets for image dithering, a change in filters, and large telescope offsets for sky background images. For all of these events, specific GeMS loops must either be paused or opened, and then resumed automatically after the event. This is handled by the SMART tools. The sequence of events for each case is described below.

Dither sequence. When a telescope offset is required, the observation sequence executor (Seqexec) sends the information to the TCS. The SMART tools then receive a message "dither" and "pause" thereby pausing all the NGS loops and dependencies (Tip-Tilt, TA, rotator, flexure and focus), while all the laser loops are kept closed. The telescope then offsets, and once all the subsystems report that they are in position, the NGS loops are resumed, the "science ready" flag is green, and the next science exposure starts. Depending on the size of the offsets, a telescope dither can take between 3 to 8 seconds. The dither pattern is set by the NGS and ODGW acquisition fields and cannot be larger than 30 arcseconds.

Filter sequence. Depending on the mode of observation, $1+3$ or $3+1$ (see section 1 ), a change in the instrument filter can have different consequences. For a $3+1$ setup (i.e. 3 Tip-Tilt NGS and 1 OI for flexure) a change in the instrument filter only implies a pause/resume of the flexure loop. In the case of fast-guiding with the GSAOI OGDWs $(1+3)$, all the NGS loops and dependencies must be paused before the filter is changed. Note that depending on the filter, the exposure time (and frame time) of the ODGW should be adjusted to keep the SNR constant. A change in filter also induces focus offsets, necessitating a shift in ODGW window positions as defined 
in a LUT. The overheads for a filter change are set by the instrument, for instance, GSAOI will take up to 30 seconds to perform any filter change.

Sky sequence. A sky sequence is somewhat similar to a dither, except that the telescope offset is usually much larger (up to 5 arcminutes). In this case, all NGS loops and dependencies are paused, and the probes are frozen and do not follow telescope offsets (as a large offset would put the probes into a hard limit if they remained in follow). We found that large telescope motions could create instabilities in the high-order loop, as the LGS may be lost for a few seconds. Because of this, we have decided to also pause the high-order loop, but keep the LGS stabilization loop closed (FSA loop).

Large telescope offsets. If the observation requires offsets larger than 5 arcminutes from the base position, the laser propagation must be stopped due to Laser Clearing House restrictions ${ }^{8}$. When the telescope returns to the original base position, the laser operator must re-acquire the LGS. Moreover, since this offset will also be unguided, the telescope operator must re-check the NGS acquisition and correct for any telescope pointing errors. In this case a separate observation block is required and extra overheads are introduced.

\subsection{Observation interruptions}

LCH Predictive Avoidance To prevent any laser illumination of sensitive satellite optics, all laser targets must first be pre-approved by the US Space Command's Laser Clearing House (LCH). A week before a laser run, Gemini provides the RA and Dec of each target and receives in return a file from the LCH showing the allowed observation windows and any times where the laser must be shuttered at that given RA and Dec. To maximize time on-sky and minimize any potential for inadvertent illumination, we have developed software that ingests the LCH file and shutters the laser 10 seconds before a window. In the current scheme however, this system is not fully automated: the astronomer needs to pause the observation while the telescope operator will open the laser loops before the shutter happens. When the shutter window has passed the laser propagation must be resumed manually. The telescope operator will re-close the laser loop and the observation sequence can then be resumed. Fortunately the NGS loop and its dependencies do not need to be paused during these shutter events since they are laser only. The current overheads associated for a LCH window are on the order of 30s 1 minute and also depends on the phasing of the window with the observation sequence. Software is currently being developed that will manage these shutter events automatically.

Aircraft Avoidance. In the case of an aircraft passing within 25 degrees of the laser propagation area, the same procedure of pause/resume as the LCH windows is used, except in this instance the laser operator must manually pause the laser propagation. While an isolated aircraft event will have a larger overhead due to the time required for an aircraft to pass through the safety zone, overall aircraft avoidance overheads are only a factor of $1.3 \mathrm{x}$ larger than satellite overheads ${ }^{8}$ as there are fewer aircraft passing near the propagation zone than satellites.

\section{CHALLENGES AND LEASSONS LEARNED}

GeMS has proven to be an exciting and challenging project. Because many complex sub-systems must work in unison, GeMS requires a level of complexity rare for an observatory facility instrument. The amount of time on sky to date (81 nights) for commissioning activity and work towards the transition into regular operations is testament alone to the complexity of the system. Because GeMS was designed over a decade ago ${ }^{26}$, the technological choices made at that time were also driven by the availability of critical components.

One example is the Gemini South laser. Today's laser technology ${ }^{27}$ and packaging will provide better sodium return ${ }^{19}$ with a much more stable system than the Gemini South laser. Achieving a satisfactory performance with the Gemini South laser, in terms of delivered power and beacon spot quality, requires a non-trivial time consuming alignment of the laser. The effort to do such had been underestimated; the laser has been the focus of extensive work for more than a year, and its commissioning has only really been considered completed as recent as May 2012.

The BTO is necessary for initial LGS constellation alignment and critical in maintaining this alignment for all telescope positions. Without a good constellation stability it is very difficult to optimize the high-order loop. With our BTO design, fulfilling the requirement on the constellation alignment and stability requires a timeconsuming fine adjustment of the optics that can only be done during nighttime. Several modifications to the 
BTO design would have made the task significantly easier ( $\mathrm{see}^{8}$ for more details).

Another lesson learned has been from the use of quad-cells for all of the GeMS WFS. Quad-cells need special on-line calibration procedures to determine the centroid gains. Errors in the centroid gains estimation will leak into the NCPA and reduce the performance. The choice of using quad-cell WFS was driven by the lack of low or zero noise detectors at that time. Using EMCCD or sCMOS detectors allows more pixels per sub-aperture without the noise trade-off. The availability now of low noise detectors also opens new design possibilities for the NGSWFS, as discussed in 2.3. Other design simplifications and hardware modifications have been discussed in a previous paper ${ }^{6}$.

The ODGW capability of GSAOI has proved to be very useful: in addition to providing Tip-Tilt information, the ODGWs can also be used as a tool to monitor performance evaluation and optimization as they provide a real-time image of the science beam as seen on the detector. This is a unique capability that can be used to provide on-line performance optimization, vibration characterization and NCPA control (ODGWs can also be used as a "truth" sensor). These optimizations are currently being done manually, but are planned to be automated in the future in order to maximize GSAOI performance.

\section{GEMS FUTURE}

With the onset of the Chilean winter, in June 2012 GeMS entered its second engineering shutdown phase. Nighttime operations are planned to resume during bright time at the end of October. Initially we will start with two runs focused on completing any outstanding commissioning tasks, and then start the System Verification (SV) in mid-December.

\subsection{Engineering Shutdown (June - October 2012)}

From June to mid-October 2012 GeMS will take advantage of the characteristically poor Chilean winter weather to be in an engineering shutdown. This shutdown period will allow for hardware fixes and upgrades based on the experience gathered on-sky, and also be used to analyze any outstanding data from previous runs. For the LGSF the shutdown plan includes a full re-optimization of the laser, improvement of the LSE temperature stability, and measuring and optimizing the BTO transmission and polarization control. For Canopus, the main focus will be the NGSWFS refurbishment as described in section 2.3. The plan also includes the commissioning of the ADC and BS and of a full RTC spare. Special effort will be devoted to documentation, software development for observation automation and the transition into operations.

\subsection{Finishing up 2012}

The end of 2012 should also bring the end of the GeMS commissioning and the beginning of science operations. The first opportunity to the community for GeMS use with GSAOI will be offered in three SV blocks for a total of $\sim 80$ hours spread over November, December and January 2013 (the end of the official Gemini 2012B semester).

\subsection{3}

The next year will be another busy one with the main focus of transitioning GeMS into regular operations. GeMS will be offered for approximately 10 nights per month, with an annual shutdown period of two months between June and August. In parallel, the second mode of operations, $1+3$ using fast ODGW for Tip-Tilt corrections, will be commissioned and offered as a science mode. A major milestone for GeMS in 2013 will be the installation and commissioning of the third DM, now being manufactured at CILAS. The DM currently being used as DM0 will re-conjugated to $4.5 \mathrm{~km}$ and the new DM will be re-integrated to be conjugated to the ground. It is expected that this upgrade will further improve GeMS performance. The new focal plane NGSWFS should also be implemented in mid-2013, which will improve sky coverage limitations. Finally, the commissioning of GeMS with Flamingos-II is also planned to start next year, with the goal to start the SV by the end of 2013. 


\subsection{GeMS long-term future}

During the March 2012 run we experienced an issue with the cooling of GSAOI. To try and maintain progression with commissioning, GeMS was used in conjunction with GMOS. Although the combination of GeMS and GMOS is not intended to be offered as a standard mode in the immediate future, this configuration proved quite useful for commissioning and verification purposes and demonstrated the capabilities of GeMS over a broad spectral range. Figure 12 shows an exciting example image of GeMS + GMOS. The performance reached was on the order of a gain of 2 to 5 in the FWHM over the natural seeing conditions. The best obtained seeing in i-band was 80 mas at FWHM, with natural seeing of $\sim 0.4$ "; the poorest GeMS corrected seeing was $\sim 350$ mas at FWHM in i-band with natural seeing of $\sim 0.9^{\prime \prime}$. The current filters which work with GeMS are: i, z and CaIII. This is limited by the beam splitter in Canopus, which cuts the light at $0.85 \mathrm{um}$ : everything below is kept for the NGSWFS. A beam splitter with a shorter cut could be installed to allow for observations in additional GMOS filters, at the cost of reduced sky coverage. A study is needed to quantify this.

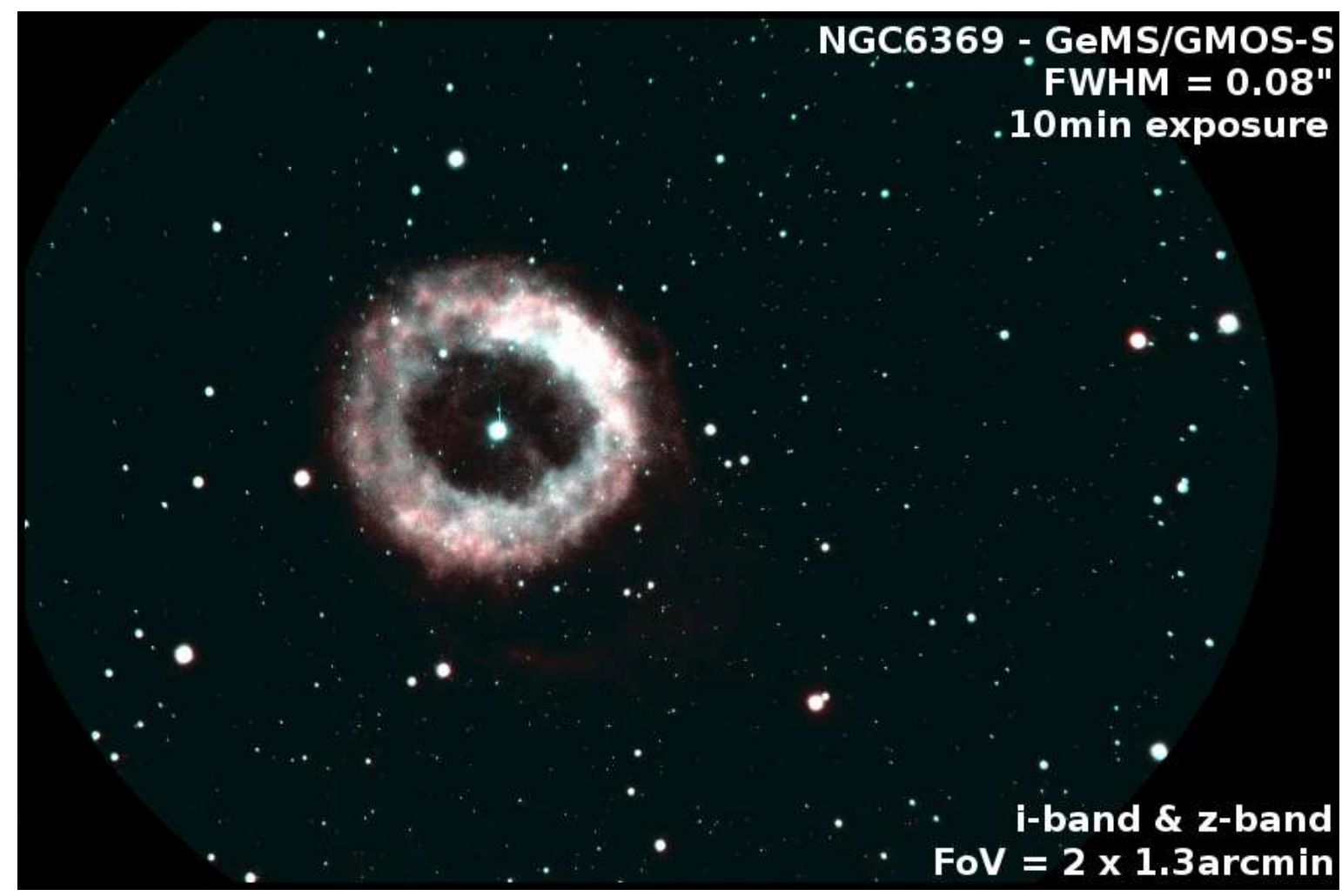

Figure 12. NGC6369 observed with GeMS + GMOS-S. The FWHM at the center of the planetary nebulae is 80 mas in iand z-band.

\section{GEMS AS A PATHFINDER}

GeMS is the first, and only, MCAO system to date using laser guide stars. In that sense, GeMS fills a special place in the AO world: it is both a testing platform and a pathfinder for future AO instrumentation. The GeMS integration and commissioning has made use of cutting edge hardware and software.

\subsection{New controllers for vibrations rejection}

While the impact on performance is relatively modest (a few \% of SR), GeMS does see vibrations. Some vibration peaks dominate, with an RMS value ranging from 5 to 10 mas. These vibrations are also seen with short GSAOI ODGW integrations. To reduce the effect of these vibrations on the performance, we have implemented and tested the use of advanced controllers for vibrations rejection. This work is done In collaboration with the 
Catolica University of Santiago, Chile and more details can be found in other papers ${ }^{28,29}$. First on-sky results are shown in Fig.13. The PSD of the turbulence in Open Lool (OL) is shown in black, a classical integrator with optimized gains is in blue, and the new controller is in red. The RMS of the Tip-Tilt respectively goes from 100mas in OL, to $31 \mathrm{mas}$ with the classical integrator and down to $27.5 \mathrm{mas}$ with the new controller. The vibration seen around $14 \mathrm{~Hz}$ is also effectively suppresed with the new method. The new controller has proven to be stable on-sky, and we are implementing an on-line adjustment of its parameters, ${ }^{30}$ which should keep the vibrations to a minimal level.

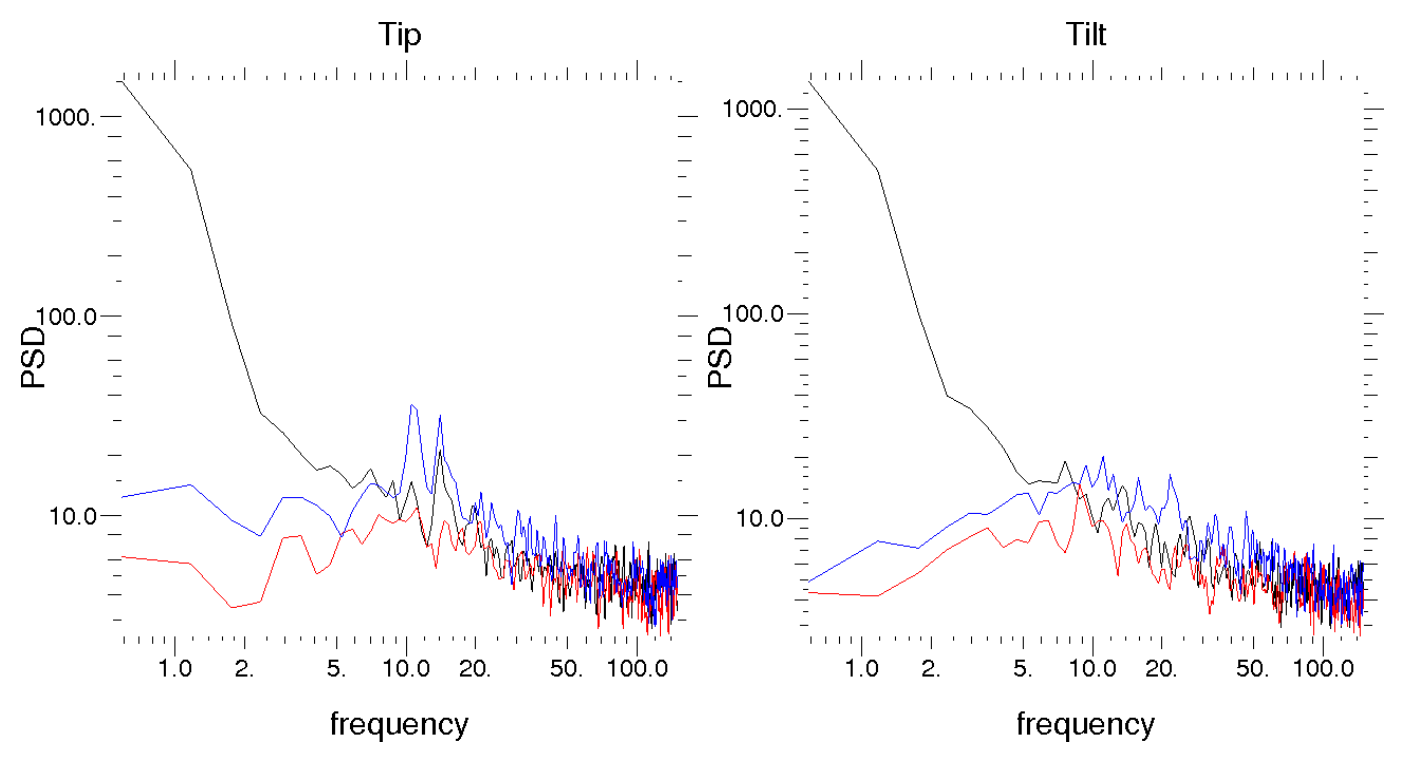

Figure 13. On-sky PSD of the residual NGSWFS Tip (left) and Tilt (right) for the full turbulence (black), the classical integrator (blue) and an advanced controller (red).

\subsection{Embedded Cn2}

Using the LGS constellation and the AO loop telemetry, we have implemented an embedded Cn2 and wind profiler. All the details about this work are found in the following papers. ${ }^{17,31}$ These parameters allow us to finely characterize GeMS performance and will be used to optimize the tomographic process on-line. Characterizing the GeMS performance in such a manner also brings useful information on the site image quality and dome seeing. The two examples shown in Fig. 14 both show a strong ground layer. The left profile is more favorable to the current two-DM configuration as most of the turbulence seen matches the altitude at which the DMs are conjugated (0 and $9 \mathrm{~km})$.

\subsection{Focal plane WFS}

We are investigating the possibility of using the ODGWs of GSAOI as a low order focal plane WFS. This is particularly interesting for GeMS, because as stated above, one of the current performance limitation is related to NCPA variations due to centroid gains mis-calibrations. Having a truth sensor capability would solve these errors. In addition, such a WFS could be used for the slow focus control, and would replance the current SFS. This would potentially improve the limiting magnitude, as all the visible light reaching the NGSWFS could then be used for Tip-Tilt sensing only. We have performed a first set of tests by using the LInearized Focalplane Technique (LIFT) ${ }^{32}$. More details on the first results are presented in a separate paper ${ }^{33}$. The original method requires to add known aberrations (e.g. an astigmatism) to the focal plane images. However, in our application, we are investigating how the residual NCPA could be used instead. Indeed, the residual NCPA cannot be completely nullified over the entire FoV, but the residual aberrations can be precisely estimated by a phase diversity method. If demonstrated, the combination of LIFT and the ODGW will provide a very powerful truth sensor, probing the aberrations in four different positions in the field. 

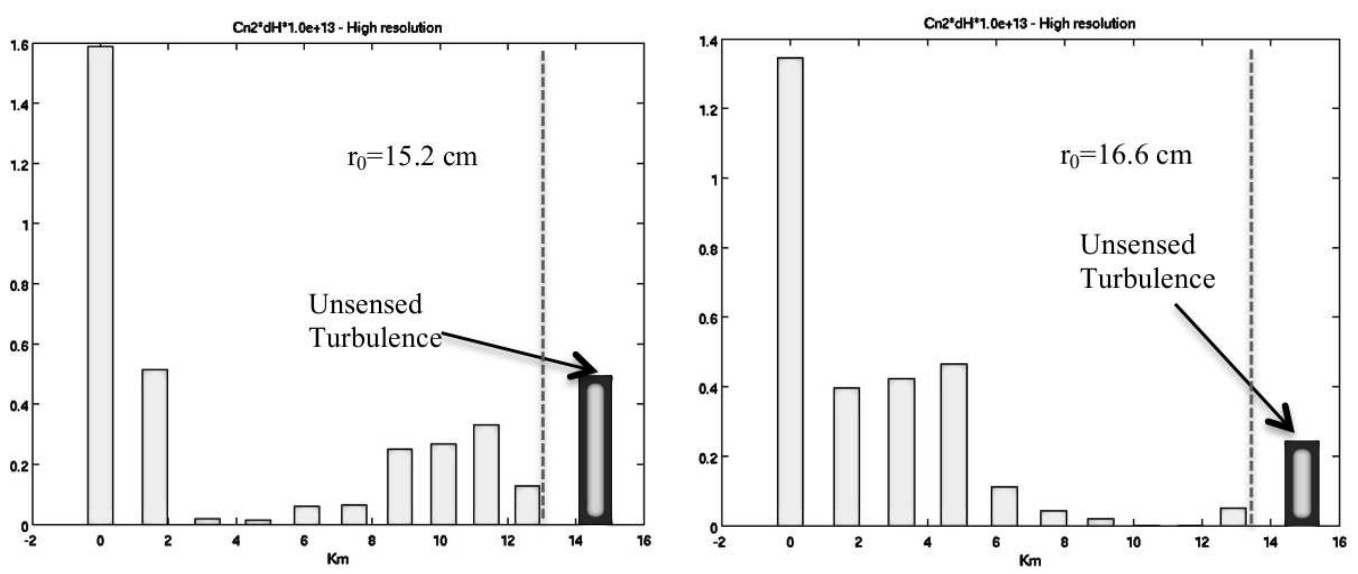

Figure 14. Example of two Cn2 profiles reconstructed from the GeMS telemetry. Unsensed turbulence is the the remaining turbulence that lies above our SLODAR detection limit. Left: night of 13/03/12 at 01:13:01. Right: night of 16/03/12 at 00:19:03.

\subsection{Numerical coronagraph}

Placing an ODGW in front of a bright star as a numerical coronagraph could avoid saturating the detector and enhance the surrounding bright star signal. A star with $\mathrm{K}=13.2$ will saturate the GSAOI detector in its minimum exposure time of $5.3 \mathrm{~s}^{16}$. Attenuating the star brightness with an ODGW allows study of the surrounding of very bright stars, without losing information as the core photometry could still be retrieved from the ODGW files.

\subsection{PSF reconstruction}

The uniformity of the PSF over the field obtained with GeMS already vastly improves the accuracy of the image analysis. Providing an estimation of the PSF across the GSAOI field would enhance the science return of MCAOcorrected images even further. Based on the method developed by Luc Gilles, ${ }^{34}$ we are starting a program to estimate the GeMS PSFs. The approach is based on a high-fidelity simulation model of the AO system. Again, the ODGWs bring a unique and valuable input for this work, providing a set of short-exposure images in different locations over the field.

\section{CONCLUSION}

GeMS is a complex but rewarding instrument. The first images have already demonstrated its scientific potential, and after more than a year of commissioning GeMS is finally close to completion and ready for science. The progress that has been made during this year is truly impressive. A dedicated effort has been made to stabilize the hardware and minimize the time lost due to technical issues. In particular, the laser and BTO are now considered as fully commissioned and part of regular operations. The transition of GeMS from commissioning to operations has also been a concentrated effort. More work is still needed, but we are confident that GeMS will be operated efficiently in the near future. More effort is required on optimizing the performance of each sub-system, and the experience gained by the team during this commissioning period is crucial to reach that objective. It is important to emphasize that no major show stoppers have been found on the way, although many challenges remain. The two remaining major challenges are the limiting laser photon return and NGSWFS sensitivity. Several options are being considered to solve the LGS flux limitation issue. One of those would be to use advanced controller for the high-order loop that would result in a better and more robust performance at low flux. This however would require major changes in the real-time control of GeMS. Another option, albeit one much more involved, would be to replace the current laser by a newer, more efficient technology. Regarding the NGSWFS, the work toward a better sensitivity has already been started, and we expect to be able to update the current hardware within a year. The first years of science operations will certainly be challenging, yet rewarding. GeMS will undoubtably be a first-class facility instrument at the Gemini South observatory. 


\section{ACKNOWLEDGMENTS}

We want to thank all the Gemini staff members who are contributing to the GeMS project, both at Gemini North and South. The Gemini Observatory is operated by the Association of Universities for Research in Astronomy, Inc., under a cooperative agreement with the NSF on behalf of the Gemini partnership: the National Science Foundation (United States), the Science and Technology Facilities Council (United Kingdom), the National Research Council (Canada), CONICYT (Chile), the Australian Research Council (Australia), Ministério da Ciência e Tecnologia (Brazil) and Ministerio de Ciencia, Tecnología e Innovación Productiva (Argentina).

\section{REFERENCES}

[1] Rigaut, F., Neichel, B., Boccas, M., and et al., "Gems: first on-sky results," in [Adaptive Optics Systems $I I I]$, Ellerbroek, B., Marchetti, E., and Veran, J.-P., eds., Proc. SPIE 8447-18 (2012).

[2] Bec, M., Rigaut, F., and et al., "The gemini mcao bench: system overview and lab integration," in [Adaptive Optics Systems], Hubin, N., Max, C., and Wizinowich, P., eds., Proc. SPIE 7015, 701568 (2008).

[3] Boccas, M., Rigaut, F., and et al., "Gems: Gemini mcao system, current status and commissioning plans," in [Adaptive Optics Systems], Hubin, N., Max, C., and Wizinowich, P., eds., Proc. SPIE 7015, 70150X (2008).

[4] D'Orgeville, C., Daruich, F., and et al., "The gemini south mcao laser guide star facility: getting ready for first light," in [Adaptive Optics Systems], Hubin, N., Max, C., and Wizinowich, P., eds., Proc. SPIE 7015, 70152P (2008).

[5] Neichel, B., Rigaut, F., and et al., "The gemini mcao system gems: nearing the end of a lab-story," in [Adaptive Optics Systems II], Ellerbroek, B. L., Wizinowich, P., Hart, M., and Hubin, N., eds., Proc. SPIE 7736, 7736 (2010).

[6] Rigaut, F., Neichel, B., and et al., "Gemini south mcao on-sky results," in [2st AO4ELT conference Adaptative Optics for Extremely Large Telescopes], Véran, J., Clénet, Y., and Fusco, T., eds., EDP Sciences 2 (2012).

[7] Cavedoni, C. P., Bombino, S., Sheehan, M., and et al., "The gemini mcao infrastructure: Laser service enclosure and support structure," in [Ground-based and Airborne Telescopes II], M., S. L. and Roberto, G., eds., Proc. SPIE 7012, 70122Y (2008).

[8] d'Orgeville, C., Neichel, B., Rigaut, F., and et al., "Gemini south multi-conjugate adaptive optics (gems) laser guide star facility on-sky performance results," in [Adaptive Optics Systems III], Ellerbroek, B., Marchetti, E., and Veran, J.-P., eds., Proc. SPIE 8447-62 (2012).

[9] http://www.gemini.edu/sciops/instruments/?q=node/10235

[10] http://www.gemini.edu/sciops/instruments/?q=node/10234

[11] http://www.gemini.edu/sciops/instruments/?q=node/10625

[12] Young, P., McGregor, P., van Harmelena, J., and Neichel, B., "Using odgws with gsaoi: Software and firmware implementation challenges," in [Adaptive Optics Systems III], Ellerbroek, B., Marchetti, E., and Veran, J.-P., eds., Proc. SPIE 8447 (2012).

[13] Neichel, B. and Rigaut, F., "Gems commissioning progress," in [Gemini Focus], 43, 45 (2011).

[14] d'Orgeville, C., "Laser first light at gemini south," in [Gemini Focus], 42, 23 (2011).

[15] Neichel, B., Rigaut, F., and et al., "Sodium photon return, spot elongation and fratricide effect: First on-sky results with gems," in [2st AO4ELT conference - Adaptative Optics for Extremely Large Telescopes], Véran, J., Clénet, Y., and Fusco, T., eds., EDP Sciences 2 (2012).

[16] Carrasco, R., Edwards, M., McGregor, P., and et al., "Results from the commissioning of the gemini south adaptive optics imager (gsaoi) at gemini south observatory," in [Adaptive Optics Systems III], Ellerbroek, B., Marchetti, E., and Veran, J.-P., eds., Proc. SPIE 8447-23 (2012).

[17] Cortes, A., Neichel, B., Guesalaga, A., and et al., "Atmospheric turbulence profiling using multiple laser star wavefront sensors," MNRAS submitted (2012).

[18] Neichel, B., Parisot, A., Petit, C., and et al., "Identification and calibration of the interaction matrix parameters for ao and mcao systems," in [Adaptive Optics Systems III], Ellerbroek, B., Marchetti, E., and Veran, J.-P., eds., Proc. SPIE 8447-209 (2012). 
[19] Neichel, B., Callingham, J., d'Orgeville, C., and et al., "Characterization of the sodium layer at cerro pachon and impact for gems performance," in [Adaptive Optics Systems III], Ellerbroek, B., Marchetti, E., and Veran, J.-P., eds., Proc. SPIE 8447-176 (2012).

[20] Feautrier, P., "A decadal survey of ao wavefront sensing detector developments in europe," in [2st AO4ELT conference - Adaptative Optics for Extremely Large Telescopes], Véran, J., Clénet, Y., and Fusco, T., eds., EDP Sciences 2 (2012).

[21] www.scmos.com

[22] Christou, J., Walls, B., Boccas, M., and et al., "Increasing sky coverage with the gemini north altair/lgs ao system," in [Adaptive Optics Systems III], Ellerbroek, B., Marchetti, E., and Veran, J.-P., eds., Proc. SPIE 8447-123 (2012).

[23] Rousset, G., Primot, J., and Fontanella, F., "Visible wavefront sensor development," LEST Foundation 28, 17-33 (1987).

[24] Nicolle, M., Fusco, T., Rousset, G., and Michau, V., "Improvement of shack-hartmann wave-front sensor measurement for extreme adaptive optics," ApOpt 29, 2743-2745 (2004).

[25] Rigaut, F., Neichel, B., and et al., "Myst: a comprehensive high-level control tool for gems," in [Adaptive Optics Systems II], Ellerbroek, B. L., Wizinowich, P., Hart, M., and Hubin, N., eds., Proc. SPIE 7736, 7736 (2010).

[26] "Gemini mcao preliminar design review," (2001).

[27] Bonaccini, D., Guidolin, I., Friedenauer, A., and et al., "The eso transportable laser guide star unit for on sky measurements of lgs photon return and other experiments," in [Adaptive Optics Systems III], Ellerbroek, B., Marchetti, E., and Veran, J.-P., eds., Proc. SPIE 8447-61 (2012).

[28] Neichel, B., Rodriguez, I., Guesalaga, A., and et al., "Kalman and h-infinity controllers for gems," in [Adaptive Optics: Methods, Analysis and Applications], OSA Technical Digest JWA32 (2011).

[29] Rodriguez, I., Neichel, B., Guesalaga, A., and et al., "Vibration characterization and mitigation at the gemini-south telescope," in [2st AO4ELT conference - Adaptative Optics for Extremely Large Telescopes], Véran, J., Clénet, Y., and Fusco, T., eds., EDP Sciences 2 (2012).

[30] Guesalaga, A., Neichel, B., Rigaut, F., and et al., "Design of frequency-based controllers for vibration mitigation at the gemini-south telescope," in [Adaptive Optics Systems III], Ellerbroek, B., Marchetti, E., and Veran, J.-P., eds., Proc. SPIE 8447-37 (2012).

[31] Cortes, A., Neichek, B., Guesalaga, A., and et al., "First results on a cn2 profiler for gems," in [2st AO4ELT conference - Adaptative Optics for Extremely Large Telescopes], Véran, J., Clénet, Y., and Fusco, T., eds., EDP Sciences 2 (2012).

[32] Meimon, S., Fusco, T., and Mugnier, L., "Lift: a focal-plane wavefront sensor for real-time low-order sensing on faint sources," Opt. Lett. 35, 3036-3038 (2010).

[33] Plantet, C., Meimon, S., Conan, J., and Fusco, T., "Lift - a noise-effective low order focal-plane sensor: from theory to full experimental validation," in [Adaptive Optics Systems III], Ellerbroek, B., Marchetti, E., and Veran, J.-P., eds., Proc. SPIE 8447-60 (2012).

[34] Gilles, L., Ellerbroek, B. L., Wang, L., and et al., "Point spread function reconstruction for laser guide star multi conjugate adaptive optics systems on extremely large telescopes," in [2st AO4ELT conference Adaptative Optics for Extremely Large Telescopes], Véran, J., Clénet, Y., and Fusco, T., eds., EDP Sciences 2 (2012). 